\title{
Eisenhart's theorem and the causal simplicity of Eisenhart's spacetime
}

\author{
E Minguzzi \\ Department of Applied Mathematics, Florence University, Via S. Marta 3, 50139 \\ Florence, Italy \\ E-mail: ettore.minguzzi@unifi.it
}

\begin{abstract}
We give a causal version of Eisenhart's geodesic characterization of classical mechanics. We emphasize the geometric, coordinate independent properties needed to express Eisenhart's theorem in light of modern studies on the Bargmann structures (lightlike dimensional reduction, pp-waves). The construction of the space metric, Coriolis 1-form and scalar potential through which the theorem is formulated is shown in detail, and in particular it is proved a one-to-one correspondence between Newtonian frames and Abelian connections on suitable lightlike principal bundles. The relation of Eisenhart's theorem in the lightlike case with a Fermat type principle is pointed out. The operation of lightlike lift is introduced and the existence of minimizers for the classical action is related to the causal simplicity of Eisenhart's spacetime.
\end{abstract}

\section{Introduction}

The mathematical study of lightlike dimensional reduction began almost unnoticed in 1929 when Eisenhart published a work [15] which drew a correspondence between the trajectories of a dynamical system on a classical configuration space $E$ (extended to include the time) and the geodesics of a higher dimensional Lorentzian manifold $M$. While this result attracted some interest, as it was going in the direction of geometrizing Newtonian mechanics as for the older Jacobi metric model [1], the nature of the projection $\pi: M \rightarrow E$ and the lightlike character of the orbits $\pi^{-1}(e), e \in E$, was not considered as an interesting ingredient of the construction 31 .

Eisenhart's results in connection with Lorentzian geometry were then overlooked and only later rediscovered following different approaches [11, 12. Indeed, even the relation between Eisenhart's result and lightlike dimensional reduction is not well known. The aim of this work is to generalize Eisenhart's theorem is such a way that it can become an integral part of present investigations on the mathematics of lightlike dimensional reduction.

It must be mentioned that the class of Eisenhart's spacetimes is quite large and includes, for instance, the plane-fronted waves considered in general relativity [13]. Any result obtained for the Eisenhart's spacetimes specializes immediately to those. Although the terminology regarding plane waves is not always uniform we may define, following [13, a plane-fronted wave as (i) a 4-dimensional spacetime which (ii) satisfies Einstein equations (most authors consider only the vacuum case) and that (iii) admits a lightlike twist-free Killing field, plus (iv) some auxiliary topological and differentiable conditions. As we shall see, without the limit on the dimension of the spacetime given 
by (i) and the condition of solving the Einstein equations (ii), this definition would be that of an Eisenhart spacetime. If the condition that (v) the Killing field is covariantly constant is added then the class of plane-fronted waves restricts itself to that of the plane-fronted waves with parallel rays, i.e. pp-waves, whereas the Eisenhart's spacetime becomes a Bargmann structure.

It seems appropriate to keep the name Eisenhart's spacetime instead of planefronted wave for the structures to be studied in this work not only for historical precedence or because of the mentioned differences, but also for a fundamental difference in the motivations. Eisenhart's spacetimes serve as a tool for investigating the relationship between classical and relativistic physics, and thus they are not meant as realistic spacetimes, rather they provide a mean for mirroring classical spacetimes into relativistic ones.

As we shall see, the Eisenhart's spacetime is interesting for two main reasons. On the one hand because it provides a bridge between relativistic and non-relativistic physics and allows to export methods and ideas from one subject to the other (see theorem [5.5). On the other hand because several physical interesting spacetimes are indeed of Eisenhart type, thus for instance, the results obtained in this work can be applied to the plane-fronted waves of general relativity.

Let $E=T \times S, T=\mathbb{R}$, be a classical $d+1$-dimensional extended configuration space of coordinates $(t, q)$. Let $a_{t}$ be a positive definite time dependent metric on $S$, and $b_{t}$ a time dependent 1 -form field on $S$. Let $\mathcal{U}(t, q)$ be a time dependent scalar field on $S$. In a coordinate chart of $S$, we shall write $a_{t}=a_{a b} \mathrm{~d} q^{a} \mathrm{~d} q^{b}, b_{t}=b_{c} \mathrm{~d} q^{c}$. Eisenhart [15] was able to show that the trajectories of a Lagrangian system with $d$ degrees of freedom

$$
L(q, \dot{q}, t)=\frac{1}{2} a_{t}(\dot{q}, \dot{q})+b_{t}(\dot{q})-\mathcal{U}(t, q),
$$

may be obtained as the projection of the geodesics of a $d+2$-dimensional manifold $M=E \times Y, Y=\mathbb{R}$, of metric [15, 31] $⿴$

$$
\mathrm{d} s^{2}=a_{t}-\mathrm{d} t \otimes\left(\mathrm{d} y-b_{t}\right)-\left(\mathrm{d} y-b_{t}\right) \otimes \mathrm{d} t-2(\mathcal{U}+k) \mathrm{d} t^{2},
$$

where $k \in \mathbb{R}$ is a constant, $y \in \mathbb{R}$ is the variable of the additional $(d+1)$ th dimension $\left(y=q^{d+1}\right)$, and $t$ is the zeroth dimension $t=q^{0}$. Different values of $k$ do not really give different spacetimes, indeed $k$ can be sent to zero through the transformations $y=y^{\prime}-k t$. Thus the chosen value of $k$ selects a coordinate system rather than a metric, and the possibility of choosing $k$ reflects the freedom in choosing the zero level for the potential. Note that $\partial / \partial y$ is a lightlike Killing vector field.

Eisenhart did not gave to the Lorentzianity $\$$ of his manifold a particular meaning, and indeed, this fact played no particular role in his analysis. Given a solution of the dynamical system $q(t)$, he set

$$
y(t)=C-\left(\frac{1}{2}+k\right) t+\int_{0}^{t} L(q, \dot{q}, t) \mathrm{d} t,
$$

where $C$ is an arbitrary constant. The trajectory $\{t, q(t), y(t)\}$ could then be regarded as a parametrization of a spacelike geodesic of the Eisenhart spacetime with respect to a natural parameter [31], $s=t$. Moreover, every solution of the Lagrangian system could be regarded in this way.

$\ddagger$ In our convention the roles of $q^{0}$ and $q^{d+1}$ are inverted with respect to [31, Book II, Sect. 11] and there is also a different choice of sign.

$\S$ The Lorentzianity of the metric follows immediately by introducing the base of 1 -forms $\omega^{0}=\mathrm{d} t$, $\omega^{a}=\mathrm{d} q^{a}, \omega^{d+1}=\mathrm{d} y+(\mathcal{U}+k) \mathrm{d} t-b$. 
A first question is whether Eisenhart's spacetime can be characterized in some coordinate independent way and whether Eisenhart's theorem admits a causal formulation so that the representing geodesics become causal rather than spacelike. An affirmative answer to the last question would give to the causal geodesics the physical interpretation of particles moving on Eisenhart's spacetime. In fact, we shall show that both questions admit an affirmative answer and that the causal version of Eisenhart's theorem is convenient from the mathematical point of view as it allows us to obtain new results on the existence of minimizers for the classical action.

The modern coordinate-independent approach to lightlike dimensional reduction is the so called Bargmann structure approach [11, 12, thus our aim will be to start with such structures or with the slight generalization considered in [25], and then to recover the manifold $E$, the space $S$, the time $t$, the metric $a_{t}$, the 1 -form $b_{t}$ and the potential $\mathcal{U}$, so as to express and study Eisenhart's theorem. We shall see that natural coordinates do not exist and that, in order to determine $b_{t}$, one has to fix the Newtonian flow (frame) on $E$. Indeed, the 1 -form $b_{t}$ will play the role of Coriolis potential, determining the rotational inertial forces of the dynamical system. The dependence of $b_{t}$ on the frame is then expected as the inertial forces depend on how the frame chosen moves on the classical spacetime $E$. We shall also prove that the flow over $E$ can be equivalently replaced by an Abelian connection on a suitable lightlike principal bundle, its curvature giving the fictitious inertial forces associated to the Newtonian frame.

The Eisenhart metric takes its simplest and most symmetric form in the case of a free particle in Euclidean space $a_{b c}=\delta_{b c}, b_{c}=0, \mathcal{U}=$ const. Remarkably, in this case the Eisenhart metric becomes the Minkowski metric. While it will be included in our analysis, the flat case is most interestingly studied by using a group theoretical approach [36]. In [36] it has been argued that ordinary shadows behave very much like classical objects living in a quotient classical spacetime thus obeying a Galilean invariant physics. Related differential geometric studies of shadows had previously appeared in [24] and [45, Th. 4.1] (see also the account [19, Sect. 5.3.2]).

The paper is organized as follows. In section 2 the spacetime geometry induced by the presence of a lightlike Killing field is studied in detail. The classical spacetime is identified with the corresponding quotient manifold. It is in this section that most of the classical physical concepts such as absolute simultaneity, absolute time, space metric, Newtonian frame and Coriolis inertial forces are introduced. In section 3 the light lift is introduced, its relation with the classical action explained and the causal version of Eisenhart's theorem is stated. In the short section 4 the Eisenhart's theorem is proved. In section 5 the Eisenhart's theorem in the lightlike case is related to a kind of Fermat's principle in the case of lightlike target curves, and the existence of minimizers for the classical action is shown to follow from the causal simplicity of Eisenhart's spacetime. Finally, in section 6 some conclusions are drawn and in the appendix a brief introduction to Newton-Cartan theory is given so as to clarify the differences in aim an motivations between this work and those on that theory.

Our notations are as follows. Let $d \in \mathbb{N}$. The indices $i, j, k$, take the values $1, \ldots, d+1$, the indices $a, b, c$, take the values $1, \ldots, d$. The Greek indices $\alpha, \beta, \mu, \nu$, take the values $0,1, \ldots, d+1$, and the indices $A, B, C$, take the values $0,1, \ldots, d$. We use the spacelike convention $\eta_{00}=-1$, and units such that $c=1$. The exterior product of two 1 -forms $\alpha, \beta$, is $\alpha \wedge \beta=\alpha \otimes \beta-\beta \otimes \alpha$. The generic point of the Lorentzian manifold $M$ is usually denoted with $x$ or $m$, that of the extended configuration space $E$ with $e$, and that of the configuration space $S$ with $q$. By lightlike, causal or timelike 
curve we always mean a regular curve. For basic results in Lorentzian geometry needed in this work and especially in the proof of theorem 5.5 the reader is referred to $[38,2,21]$.

\section{Lightlike Killing vector fields}

In this section we study the geometry induced on spacetime by the presence of a lightlike Killing vector field (for related investigations see [32, 48]). As we shall see a lightlike Killing field allows one to construct a quotient manifold $E$ in which the physics is Newtonian provided the physics on the original manifold $M$ was Einstenian. We shall not go into all the details which stay at the heart of this correspondence between general relativity and the so called Newton-Cartan theory on the quotient spacetime [29. The reader is referred to [11, 30, 12, 5, 25, for more on this subject. As a contribution, using coordinate independent methods, we shall prove the equivalence between Newtonian flows on the quotient manifold and Abelian connections.

Consider a $(d+1)+1$-dimensional Lorentzian spacetime $(M, g)$ with a future directed lightlike Killing vector field $n, n_{\mu ; \nu}+n_{\nu ; \mu}=0$. The previous equation implies that the field $n$ is geodesic $n_{\nu ; \mu} n^{\mu}=0$, divergenceless $n_{; \mu}^{\mu}=0$ and 'shear free' by definition. By [3, Lemma 3.2], $n$ is nowhere vanishing. We introduce an equivalence relation between events that lie on the same geodesic integral line, and denote with $E$ the quotient space $\pi: M \rightarrow E$. The set $E$ will also be called the extended configuration space, or the classical spacetime.

Now we have to add some conditions to make $E$ a $(d+1)$-dimensional manifold and $\pi$ a differentiable projection. Unfortunately, even if $n$ is a complete vector field and $M$ is a causal spacetime $E$ may not be a manifold. Indeed, there are pathological examples in which the geodesic integral lines of $n$ come arbitrarily close to themselves, preventing $E$ from being a manifold. An example can be constructed from $2+1$ Minkowski spacetime $(\Lambda, \eta), \eta=\mathrm{d} q^{2}-\mathrm{d} t \otimes \mathrm{d} y-\mathrm{d} y \otimes \mathrm{d} t-\mathrm{d} t^{2}$ by making the identifications $(t, q, y) \sim(t, q+1, y)$, and $(t, q, y) \sim(t, q+\alpha, y+1)$ where $\alpha$ is an irrational number.

One could try to impose some stronger causality constraints on $(M, g)$ such as the distinguishing or strong causality properties, however they would be too restrictive for later applications.

Thus we simply assume that $E$ is a manifold, that $\pi$ is differentiable and that $M$ has locally a direct product structure, i.e. $\mathcal{A} \times \mathbb{R}, \mathcal{A}$ open set on $E$. In other words we assume that $M$ is a principal bundle with structure group $(\mathbb{R},+)$ and fiber diffeomorphic to $\mathbb{R}$. In particular $n$ is a complete vector field. As the fiber is contractible, global sections exist [41] and hence this local assumption implies that $M$ is diffeomorphic to $E \times \mathbb{R}$. We shall refer to these properties by saying that $(M, g)$ has a principal bundle structure.

By $n$ we denote, depending on the context, the Killing vector field or the 1-form field obtained by lowering an index with $g, n_{\mu} \mathrm{d} x^{\mu}$.

Our next assumption is that the 1-form field $n$ determines a distribution of null hyperplanes, $\operatorname{ker} n$, which is locally integrable, that is

$$
n \wedge \mathrm{d} n=0 .
$$

\| The manifold is at least $C^{k}$, and the metric is at least $C^{k-1}, k=3$, but $k=2$ will suffice almost everywhere unless in those parts where the existence and continuity of the Riemann tensor is invoked. The tensor fields $a_{t}, b_{t}, U$ and $\psi$ (to be introduced later) have the same degree of differentiability of the metric. 
These requirements are weaker that those needed to define a Bargmann structure [1, 12, since there $n$ must be covariantly constant, $n_{\mu ; \nu}=0$, and hence closed $\mathrm{d} n=0$.

The following theorem, which clarifies earlier results 13, 25] (see also [22]), relates the hypersurface orthogonality condition (4) with an Einstein equation on $M$.

Theorem 2.1. Let $n$ be a lightlike Killing vector field on the spacetime $(M, g)$. In any spacetime dimension $n \wedge \mathrm{d} n=0$ if and only if $R_{\mu \nu} n^{\mu} n^{\nu}=0$. In particular if the spacetime has dimension lower than 3 then $n \wedge \mathrm{d} n=0$, and $R_{\mu \nu} n^{\mu} n^{\nu}=0$ whereas if $M$ is 4-dimensional $(d=2)$, then $R_{\mu \nu} n^{\mu} n^{\nu} \geq 0$ and

$$
z_{\alpha}:=2 \epsilon_{\alpha \beta \gamma \delta} n^{\beta} n^{[\gamma ; \delta]}= \pm\left(8 R_{\mu \nu} n^{\mu} n^{\nu}\right)^{1 / 2} n_{\alpha},
$$

where the sign is constant and well defined in those disconnected open sets where $R_{\mu \nu} n^{\mu} n^{\nu}>0$.

Proof. From $n_{\beta ; \alpha ; \mu}-n_{\beta ; \mu ; \alpha}=R_{\beta \nu \mu \alpha} n^{\nu}$, and permuting repeatedly the indices and summing and subtracting the equations so obtained we have (Killing vector lemma) $n_{\beta ; \alpha ; \mu}=-R_{\mu \nu \alpha \beta} n^{\nu}$. Thus

$$
n^{\alpha ; \beta} n_{\alpha ; \gamma}=\left(n^{\alpha} n_{\alpha ; \gamma}\right)^{; \beta}-n^{\alpha} n_{\alpha ; \gamma} ; \beta=R_{\nu \gamma \alpha}^{\beta} n^{\nu} n^{\alpha},
$$

In particular $n_{[\alpha ; \mu]} n^{[\alpha ; \mu]}=R_{\mu \nu} n^{\mu} n^{\nu}$. Note that $n^{\mu} n_{\mu}=0=n_{[\alpha ; \mu]} n^{\mu}$.

Let

$$
w_{\gamma \mu \nu}=n_{\gamma} n_{[\mu ; \nu]}+n_{\nu} n_{[\gamma ; \mu]}+n_{\mu} n_{[\nu ; \gamma]},
$$

so that $n \wedge \mathrm{d} n=0$ iff $w_{\gamma \mu \nu}=0$. The tensor $w_{\gamma \mu \nu}$ is skew-symmetric. Note that $w_{\gamma \mu \nu} n^{[\mu ; \nu]}=n_{\gamma}\left(R_{\mu \nu} n^{\mu} n^{\nu}\right)$, thus $n \wedge \mathrm{d} n=0$ implies $R_{\mu \nu} n^{\mu} n^{\nu}=0$. For the converse, let $R_{\mu \nu} n^{\mu} n^{\nu}=0$, note that $w_{\alpha \mu \nu} w^{\beta \mu \nu}=n_{\alpha} n^{\beta}\left(R_{\mu \nu} n^{\mu} n^{\nu}\right)=0$. Let $\left\{x^{\mu}\right\}$ be coordinates at the chosen arbitrary event $x$ (or introduce a non-holonomic orthonormal frame) so that $g_{\mu \nu}=\eta_{\mu \nu}$ at that event. Then $0=w_{0 \mu \nu} w^{0 \mu \nu}=-\sum_{\mu, \nu \neq 0}\left(w_{0 \mu \nu}\right)^{2}$ hence $w_{0 \mu \nu}=0$. The equation $w_{\alpha \mu \nu} w^{\alpha \mu \nu}=0$, now reads $\sum_{\alpha, \mu, \nu \neq 0}\left(w_{\alpha \mu \nu}\right)^{2}=0$ and thus all the components of $w_{\alpha \mu \nu}$ vanish, i.e. $n \wedge \mathrm{d} n=0$.

Let us consider some particular cases. If the dimension of the spacetime is lower or equal to 2 then $n \wedge \mathrm{d} n=0$ follows for dimensional reasons. If the spacetime has dimension 3 defined $z=\epsilon_{\beta \gamma \delta} n^{\beta} n^{[\gamma ; \delta]}$, we have $z^{2}=-\delta_{\eta \alpha \beta}^{\delta \mu \nu} n^{\eta} n_{\delta} n^{[\alpha ; \beta]} n_{[\mu ; \nu]}=0$.

If the spacetime has dimensions 4 then the following quantity vanishes

$$
z_{\sigma} z^{\sigma}=-4 \delta_{\eta \alpha \beta}^{\delta \mu \nu} n^{\eta} n_{\delta} n^{[\alpha ; \beta]} n_{[\mu ; \nu]}=0 .
$$

Since $z^{\alpha}$ is a lightlike vector and $z_{\alpha} n^{\alpha}=0$, it follows that $z^{\alpha}$ must be proportional to $n^{\mu}, z^{\alpha}=f n^{\alpha}$. The identity

$$
\begin{aligned}
f^{2} n_{\mu} & =f z_{\mu}=2 \epsilon_{\mu \nu \alpha \beta} z^{\nu} n^{[\alpha ; \beta]}=4 \delta_{\mu \alpha \beta}^{\gamma \eta \sigma} n_{\gamma} n_{[\eta ; \sigma]} n^{[\alpha ; \beta]} \\
& =8 n_{\mu}\left(n_{[\alpha ; \beta]} n^{[\alpha ; \beta]}\right)=\left(8 R_{\alpha \beta} n^{\alpha} n^{\beta}\right) n_{\mu},
\end{aligned}
$$

shows that $R_{\alpha \beta} n^{\alpha} n^{\beta} \geq 0$ and $f= \pm\left(8 R_{\mu \nu} n^{\mu} n^{\nu}\right)^{1 / 2}$ where, by continuity, the sign \pm is constant in those regions where $R_{\mu \nu} n^{\mu} n^{\nu}>0$. 
This result shows that the single Einstein's vacuum equation $R_{\mu \nu} n^{\mu} n^{\nu}=0$ in the spacetime $(M, g)$ is equivalent to the hypersurface 'orthogonality' of the lightlike Killing vector field.

The problem of reducing the Einstein equations to $E$ has been studied in generality in 25. Some other results will be mentioned in what follows. We do not impose the full Einstein's equations on $M$ because they would lead to a quite restrictive geometry on $E$.

\subsection{Absolute simultaneity, absolute time, Newtonian frames and space metric}

Due to Eq. (4), by Frobenius theorem there are local functions $\psi>0$ and $t$ such that $n=-\psi \mathrm{d} t$. In principle the construction that follows could be developed locally, but in order to simplify the analysis and the notation we shall assume that the distribution of hyperplanes $\operatorname{ker} n$, is not only locally integrable, but also globally integrable, that is, there are global functions $\psi>0$ and $t$ such that $n=-\psi \mathrm{d} t$. If $M$ is simply connected this condition is automatically satisfied by a Bargmann structure (as $\mathrm{d} n=0$ ).

It is time to pause and to recall all the assumptions that will not be reminded in what follows. $M$ is a Lorentzian time oriented manifold (i.e. a spacetime) endowed with a complete future directed lightlike Killing vector field $n$, which makes $(M, g)$ a principal bundle of base $E$, and whose associated distribution of hyperplanes $\operatorname{ker} n$ is globally integrable.

The function $t$ is such that given a future directed timelike vector $V$, it is $\mathrm{d} t[V]=-g(n, V) / \psi>0$, because $n$ and $V$ are in the same forward light cone. Thus $t$ strictly increases over timelike curves, but it is constant on the null geodesics generated by $n$ and in particular the submanifolds $t(x)=\bar{t}$ are null hypersurfaces. The function $t$ satisfies the requirements for a quasi-time function in the sense of [2, Def. 13.4], nevertheless we shall call it simply time function not because of its role on $M$ but because of the role that it will play on $E$ (see figure 1). Indeed, $\mathrm{d} t[n]=\partial_{n} t=0$ so that $t$ is a function on $E$ lifted to $M$. We shall denote with the same letter $t$ the time function on $E$ and its lift to $M$. The condition $L_{n} n=0$ implies $\partial_{n} \psi=0$, thus $\psi$ depends only on the base point too.

The global integrability condition implies the existence of a natural absolute simultaneity on $E$, determined by the surfaces $t=$ cnst. However, note that the time function is not, by itself, completely determined since one can change time function $t$ to $t^{\prime}=f(t), f^{\prime}>0$, by suitably changing $\psi: E \rightarrow \mathbb{R}$. For this reason, in what follows, we shall always keep the field $\psi$ aside a time differential, for instance in expressions like $\psi \mathrm{d} t$ or (when it will make sense) $\frac{1}{\psi} \frac{\partial}{\partial t}$.

Each null hypersurface $N_{\bar{t}}$ on $M$, of equation $t(x)=\bar{t}$, may be regarded as a principal bundle over $S_{\bar{t}}$ (i.e. the locus $t(x)=\bar{t}$ on $E$ ) with fiber $\mathbb{R}$ and structure group $(\mathbb{R},+)$ generated by $n$.

If we are in a Bargmann structure, $\mathrm{d} n=0$, then $\psi$ depends solely on $t$, and we can choose the new time parameter $t^{\prime}, \mathrm{d} t^{\prime}=\psi \mathrm{d} t$, so that $\psi \rightarrow 1$. We conclude that a Bargmann structure has a natural absolute time on $E$, i.e. the one that makes $\psi=1$.

Let $m \in M$ and $e=\pi(m) \in E$. The projection $\pi$ is a submersion, that is for any tangent vector $v$ at $e$ there is a tangent vector $V$ at $m$ that projects on it, $v=\pi_{*} V$. Moreover, $n$ spans the kernel (vertical space) of this projection. Any tangent vector

$v$ at $e$ can therefore be represented in the full spacetime with the equivalence class $[V]$, where $V \in T M_{m}$ is a representative and any alternative representative reads $V^{\prime}=V+\alpha n$ for a suitable $\alpha \in \mathbb{R}$. 


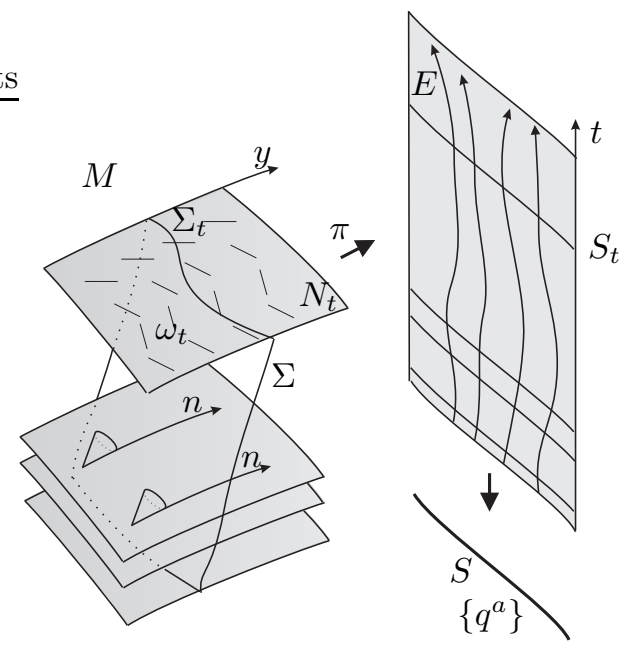

Figure 1. The spacetime $(M, g)$ due to the condition $n \wedge \mathrm{d} n=0$ on the lightlike Killing field $n$ splits into principal bundles $\pi_{t}: N_{t} \rightarrow S_{t}$. A $t$-parameter family of connection 1-forms $\omega_{t}$ on those bundles (displayed here with their kernel on the tangent spaces $T N_{t}$ ) is equivalent to a Newtonian extended frame on $E$. The connections $\omega_{t}$ have in general an holonomy which, unfortunately, can not be displayed in this figure since $S$ here is one-dimensional.

In order to construct coordinates on $M$ we first need natural coordinates on $E$.

Definition 2.2. By Newtonian extended reference frame, or simply Newtonian frame, we mean a future directed (at least $C^{1}$ ) vector field $w(e)$ on $E$ (future directed means $\mathrm{d} t[w]>0$ ) normalized in such a way that $\psi \mathrm{d} t[w]=1$.

In $M$ the Newtonian frame is represented by a field of equivalence classes $[W(m)]$ where $W(m)$ is a representative vector field such that $g(n, W)=-1<0$ (any representative field satisfies this relation).

The integral lines of $w(e)$ on $E$ form a $d$-dimensional quotient space $S$ that we call the space of the reference frame. We cover $S$ with charts of coordinates denoted $q^{a}$, and $\left(t, q^{a}\right)$ become coordinates on $E$. We stress that natural coordinates (up to transformations $\left.q^{\prime a}=q^{\prime a}\left(q^{b}\right)\right)$ on $E$ are given only if a choice of Newtonian frame has been made. In these coordinates the normalized vector field that defines the frame is $w=\frac{1}{\psi} \frac{\partial}{\partial t}$ (one should be careful because this expression makes sense on $E$ but it does not make sense on $M$ since the last coordinate $y$ has not yet been defined). The Newtonian frame gives another fibration $\pi_{E S}: E \rightarrow S$, and the quotient space $S$ can also be identified with any chosen slice $S_{t}, t=$ cnst. on $E$. We have $E=T \times S$ where $T$ is the image of the function $t: E \rightarrow \mathbb{R}$. It is an open connected subset of $\mathbb{R}$ which can be different from $\mathbb{R}$, as it also depends on the choice of $\psi$. In the case of a Bargmann structure, as $t$ is uniquely determined, it is natural to ask that $T=\mathbb{R}$.

The metric $g$ induces a (Newtonian frame independent) Riemannian metric on $S_{t}$ as follows. Let $w, v$ be tangent vectors to $S_{t}$ at $q \in S_{t}$, then define the Riemannian metric

$$
a_{t}(w, v)=g(W, V),
$$

where $W$ and $V$ are any representatives on $N_{t}$. The right hand side is independent of the representatives, since as $w$ and $v$ are tangent to $S_{t}$ we have $V^{\mu} n_{\mu}=W^{\mu} n_{\mu}=0$, 
i.e. $V$ and $W$ are tangent to $N_{t}$. The condition $a_{t}(v, v)=0$ implies $g(V, V)=0 \Rightarrow$ $V \propto n \Rightarrow v=0$, that is $a_{t}$ is positive definite. In a coordinate chart $\left(t, q^{a}\right)$ adapted to the Newtonian frame the metric on $S_{t}$ may be written

$$
a_{t}(w, v)=a_{a b}(t, q) \mathrm{d} q^{a} \mathrm{~d} q^{b} .
$$

Note that while the space $S$ is a Newtonian frame dependent concept, the spaces $S_{t}$ endowed with the metrics $a_{t}$ are not. Due to the identification of the different $S_{t}$ on a single space $S$ provided by the Newtonian reference frame, the metric $a_{t}$ can also be regarded as a time dependent metric on $S$.

\subsection{Equivalence between Newtonian frames and Abelian connections}

Interestingly, the Newtonian frame $v(e)$ determines a connection (in fact a oneparameter family, one for each choice of $t$ ) over the principal bundle $\pi_{t}: N_{t} \rightarrow S_{t}$ $\left(\pi_{t}:=\left.\pi\right|_{N_{t}}\right)$. Indeed, let $V_{t}$ be a representative vector field restricted to $N_{t}, V_{t}=\left.V\right|_{N_{t}}$, and construct the 1-form $\omega_{t}(\cdot)=-\left.g\left(\cdot, V_{t}\right)\right|_{N_{t}}$ which acts on the vectors tangent to $N_{t}$. It is easy to see that if $W$ is tangent to $N_{t}$ at $m, n_{\mu} W^{\mu}=0$, then $\omega_{t}(W)$ is independent of the representative $V_{t}$ chosen, and depends only on the Newtonian frame at time $t$. Moreover, the following properties can be easily checked taking into account that, by construction, $L_{n} V \propto n$,

(a) $\omega_{t}(n)=1$,

(b) $L_{n} \omega_{t}=0$.

Thus, $\omega_{t}$ is a connection 1-form on $\pi_{t}: N_{t} \rightarrow S_{t}$, [26. One should be careful because $\omega_{t}$ is not defined on vectors which are not tangent to $N_{t}$.

Conversely, any $\left(C^{1}\right)$ one-parameter family of connections $\omega_{t}$ on the principal bundles $\pi_{t}: N_{t} \rightarrow S_{t}$ is associated to a Newtonian frame on $E$. Since the proof is somewhat more involved we give it in the form of a theorem.

Theorem 2.3. Let $v(e)$ be a future directed vector field on $E$ such that $\psi \mathrm{d} t[v]=1$ (Newtonian frame), and let $V(m)$ any vector field on $(M, g)$ which projects on it, then $\omega_{t}(\cdot)=-\left.g(\cdot, V)\right|_{N_{t}}\left(\right.$ a 1-form which acts on $\left.T N_{t}\right)$ is a connection for the principal bundle $\pi_{t}: N_{t} \rightarrow S_{t}$ which is independent of the representative $V(m)$.

Conversely, let $\omega_{t}$ be a $\left(C^{1}\right)$ one-parameter family of connections for the principal bundles $\pi_{t}: N_{t} \rightarrow S_{t}$ (i.e. 1-form fields over $N_{t}$ which satisfy (a) and (b) above). There is a unique Newtonian frame $v(q)$ on $E$ such that for any representative field $V, \pi_{*} V=v$, it is $\omega_{t}(\cdot)=-\left.g(\cdot, V)\right|_{N_{t}}$.

Proof. The first part has been already proved. Introduce an arbitrary vector field $W$ on $M$ such that $-g(n, W)>0$. Extend $\omega_{t}$ to a 1-form field $\omega$ over $M$ such that, (i) $\omega_{t}(\bar{W})=\left.\omega(\bar{W})\right|_{N_{t}}$, for every $\bar{W}$ such that $n_{\mu} \bar{W}^{\mu}=0$, i.e. the restriction to the manifold $N_{t}$ coincides with $\omega_{t}$ and, (ii) $\omega(W)=0$. Since $g$ is invertible there is a unique vector field $V$ such that $\omega(\cdot)=-g(\cdot, V)$. The field $V$ is not tangent, at any point, to $N_{t}$, indeed $n_{\mu} V^{\mu}=-\omega[n]=-\omega_{t}[n]=-1 \neq 0$. Moreover, condition (b) implies that $L_{n} V=0$, hence $V$ is projectable on a vector field $\pi_{*} V=v$ on $E$ such that $\psi \mathrm{d} t[v]=1$. Note that by changing $V \rightarrow V+\alpha(m) n$, the 1 -form $-g(\cdot, V)$ restricted to the manifold $N_{t}$ still coincides with $\omega_{t}$. Now, let us show that the choice of $W$ does not change the field $v$. The reason is that if $W \rightarrow W^{\prime}$, then $V \rightarrow V^{\prime}$ where

$$
V^{\prime}=V-\frac{g\left(V, W^{\prime}\right)}{g\left(n, W^{\prime}\right)} n .
$$




\begin{tabular}{|c|c|}
\hline \multicolumn{1}{|c|}{ Gauge theory } & Physical interpretation \\
\hline \hline $\begin{array}{c}\text { Lightlike Killing field } n \text { on }(M, g) . \\
\text { Principal bundle } \pi: M \rightarrow E\end{array}$ & $\begin{array}{c}\text { Existence of a quotient space } E \\
\text { the Newtonian spacetime) }\end{array}$ \\
\hline $\begin{array}{c}n \wedge \mathrm{d} n=0 . \text { One-parameter family } \\
\text { of principal bundles } \pi_{t}: N_{t} \rightarrow S_{t}\end{array}$ & $\begin{array}{c}\text { Absolute simultaneity on } E . \quad \text { A } \\
\text { particular vacuum Einstein equa- } \\
\text { tion on } M \text { is satisfied }\end{array}$ \\
\hline Bargmann structure, $\mathrm{d} n=0$ & Absolute time on $E$ \\
\hline Connection $\omega_{t}$ & Newtonian frame on $E$ \\
\hline Curvature $\Omega_{t}$ & Coriolis inertial forces \\
\hline$\Omega_{t}=0$, flat bundle. & Non-rotating Newtonian frame. \\
\hline
\end{tabular}

We conclude that the choice of $W$ which serves to extend $\omega_{t}$ fixes only the vertical part of $V$ and does not affect its projection.

In summary, we proved that there is a one-to-one correspondence between Newtonian frames on $E$ and one-parameter families of connections $\omega_{t}$ on the principal bundles $\pi_{t}: N_{t} \rightarrow S_{t}$.

Note that if a Newtonian frame on $E$ reads $w=\frac{1}{\psi} \frac{\partial}{\partial t}$, then one of its representative $W$ on $M$, takes the same form $W=\frac{1}{\psi} \frac{\partial}{\partial t}$ irrespective on how the coordinate $y$ is defined on $M$ (provided $n=\partial / \partial y$ ).

Each manifold $N_{t}$ has a natural exterior differential $\mathrm{d}_{t}$. The distinction between $\mathrm{d}_{t}$ and d defined over $M$ must be kept in mind. In short $\mathrm{d}_{t}$ does not differentiate with respect to the $t$ function. For instance $f(t)$ has a non-vanishing exterior differential $\mathrm{d} f \neq 0$ but $\mathrm{d}_{t} f=0$, because on the manifold $N_{t}$, the function $f$ is constant.

We can now define the curvature $\Omega_{t}=\mathrm{d}_{t} \omega_{t}$ on each principal bundle $\pi_{t}: N_{t} \rightarrow S_{t}$. The proved characterization of Newtonian frames allows us to define the non-rotating Newtonian frames as those associated with a flat connection, $\Omega_{t}=0$. Indeed, the curvature $\Omega_{t}$ will be strictly linked to the Coriolis inertial forces.

\subsection{The Coriolis potential and the Eisenhart metric}

Consider the image $\Sigma$, of a $\left(C^{1}\right)$ section $\sigma: E \rightarrow M$ (global sections exist since the fiber is contractible [41]), and define the function $y: M \rightarrow \mathbb{R}$, as the unique function such that $y=0$ over $\Sigma$, and $\mathrm{d} y[n]=1$. Because $n$ is complete, the image of $y$ is all $\mathbb{R}$ and hence $M=E \times \mathbb{R}=T \times S \times \mathbb{R}$.

Let $\pi_{S}=\pi_{E S} \circ \pi, \pi_{S}: M \rightarrow S$. Given a section $\sigma$, and a coordinate chart $\left\{q^{a}\right\}$ defined on an open set $B \subset S$, the natural coordinates on $\pi_{S}^{-1} B \subset M$, are then $\left\{x^{\mu}\right\}=\left\{t, q^{1}, \ldots, q^{d}, y\right\}$.

The section $\sigma$ can also be regarded as a 1-parameter family of sections $\sigma_{t}$ of the bundles $\pi_{t}: N_{t} \rightarrow S_{t}$, or, using the diffeomorphism between $S$ and $S_{t}$ provided by the Newtonian frame, as a 1-parameter family of sections $\sigma_{t}: S \rightarrow N_{t}$ of the 1-parameter family of principal bundles $\pi_{t}: N_{t} \rightarrow S$. 
Thus over $S$ we can define not only the time dependent metric $a_{t}$, but also the time dependent 1-form field

$$
b_{t}=-\sigma_{t}^{*} \omega_{t},
$$

which is the (minus) potential of the connection $\omega_{t}$ on the principal bundle $\pi_{t}: N_{t} \rightarrow$ $S$. If $a_{t}$ and $b_{t}$ are known to be independent of $t$ we shall omit the index $t$. The corresponding curvature reads in coordinates $\mathrm{d}_{t} b=\left(\partial_{[a} b_{b]}\right) \mathrm{d} q^{a} \wedge \mathrm{d} q^{b}=-\sigma_{t}^{*} \Omega_{t}$.

We claim that there is a time dependent function $U: E \rightarrow \mathbb{R}$, such that the metric $g$ on $M=E \times \mathbb{R}=T \times S \times \mathbb{R}$ reads (recall that $n=-\psi \mathrm{d} t$ )

$$
g=\pi_{S}^{*} a_{t}+n \otimes\left(\mathrm{d} y-\pi_{S}^{*} b_{t}\right)+\left(\mathrm{d} y-\pi_{S}^{*} b_{t}\right) \otimes n-(2 U+1) n^{2},
$$

where $\pi_{S}^{*}$ is the pullback of covariant tensors from $S$ to $M$, and where for simplicity the pullback of functions from $E$ to $M$ does not receive a different symbol (thus, as done with functions $\psi$ and $t$, we replaced $\pi^{*} U$ with $U$ ). Note that $\omega_{t}$ is undefined over vectors which are not tangent to $N_{t}$, on the contrary $\pi_{S}^{*} b_{t}$ is perfectly meaningful. This metric reduces to that considered by Eisenhart in the case of a Bargmann structure. The generalized form with $\psi$ not constant was introduced in [31, again as a pure coordinate dependent definition.

The particular form $-(2 U+1)$ for the coefficient in front of $(-\psi \mathrm{d} t)^{2}$ is chosen in order to obtain a nice Minkowskian limit for $U=0, S=\mathbb{E}^{3}, a_{t a b}=\delta_{a b}, b=0, \psi=1$, with the usual shadow coordinates (see [36]).

In order to prove Eq. (13) let us first consider two vectors $V$ and $W$ at $m \in M$, tangent to $N_{t}$ so that only the first term in the right-hand side of Eq. (13) does not vanish. We have (due to the identification between $S$ and $S_{t}, \pi_{S *}$ becomes $\pi_{*}$ )

$$
\pi_{S}^{*} a_{t}(V, W)=a_{t}\left(\pi_{*} V, \pi_{*} W\right)=g(V, W),
$$

where in the last step we used the fact that $V$ is a representative for $\pi_{*} V$, and analogously for $W$. Thus the right-hand side of Eq. (13) restricted to the vectors tangent to $N_{t}$ coincides indeed with the metric $g$.

We are now going to show that there is a vector field $W$ such that the scalar product of $W$ with a vector tangent to $N_{t}$ made with the right-hand side of Eq. (13) coincides with that made with the metric. Let $w$ be the Newtonian frame field that defines $S, \psi \mathrm{d} t[w]=1$, and let $W$ be the unique representative, $\pi_{*} W=w$, such that $\mathrm{d} y[W]=0$ (it can be obtained by Lie transporting $\sigma_{*} w$ over the fibers). Clearly, $\pi_{S *} W=0$ and $n[W]=-1$. Thus if $V$ is tangent to $N_{t}$ the scalar product made with the right-hand side of Eq. (13) reads

$$
-\left(\mathrm{d} y-\pi_{S}^{*} b_{t}\right)[V]=-\left(\mathrm{d} y[V]-b_{t}\left[\pi_{*} V\right]\right)=-\omega_{t}[V]=g(W, V) .
$$

The function $U$ is then chosen such that $g(W, W)=-(2 U+1)$ which proves the claim. The proof also shows that $\left(\mathrm{d} y-\pi_{S}^{*} b_{t}\right)$ is an extension of the connection 1-form $\omega_{t}$, in the sense that $\omega_{t}$ can be regarded as the restriction of $\left(\mathrm{d} y-\pi_{S}^{*} b_{t}\right)$ to the vectors tangent to $N_{t}$.

Given the definition

Definition 2.4. Let $S$ be a $d$-dimensional manifold endowed with a (all possibly time dependent and at least $C^{1}$ ) positive definite metric $a_{t}$, 1-form field $b_{t}$ and scalar fields $U$ and $\psi>0$. Let $T$ be a connected open subset of $\mathbb{R}$ and set $M=T \times S \times \mathbb{R}$, where $x=(t, q, y)$ is the generic event of $M$ and define $n=-\psi \mathrm{d} t$ on $M$. The Eisenhart spacetime is the manifold $M$ endowed with the metric (13) and time orientation such that $n^{\mu} \partial / \partial x^{\mu}$ is future directed. 
the results obtained can be expressed, somewhat synthetically, as follows

Theorem 2.5. Let $(M, g)$ be $a(d+1)+1$-dimensional spacetime endowed with $a$ complete lightlike Killing vector field $n$ which makes $(M, g)$ a $(\mathbb{R},+)$ principal bundle and such that the distribution of hyperplanes, kern, is globally integrable. Let $N_{t}$ be the corresponding foliation with $n=-\psi \mathrm{d} t, \psi>0, T$ the image of $t$, and let $S_{t}$ be the quotient spaces under the action of $n$, so that $\pi_{t}: N_{t} \rightarrow S_{t}$ are principal $(\mathbb{R},+)$ bundles. Let $\omega_{t}$ be a $\left(C^{1}\right)$ 1-parameter family of connections on the bundles and let $S$ be the space obtained identifying the different $S_{t}$ through the action of the Newtonian frame associated to $\omega_{t}$. Over $S$ a time dependent space metric $a_{t}$, can be defined through Eq. (9) and, given a (C $\left.C^{1}\right)$ 1-parameter family of sections $\sigma_{t}: S_{t} \rightarrow N_{t}$, a time dependent 1-form field $b_{t}$ can be defined through $E q$. (12). Then $M=T \times S \times \mathbb{R}$ and a function $U$ exists such that the metric $g$ takes the form (13). The spacetime $(M, g)$ is then a Eisenhart spacetime.

A change of section (gauge transformation) $\sigma \rightarrow \sigma^{\prime},\left(\sigma_{t} \rightarrow \sigma_{t}^{\prime}\right)$ reads

$$
\begin{aligned}
& y^{\prime}=y+\alpha(t, q), \\
& b_{t}^{\prime}=b_{t}+\mathrm{d}_{t} \alpha, \\
& \psi U^{\prime}=\psi U-\partial_{t} \alpha,
\end{aligned}
$$

and $\omega_{t}$ is invariant as expected. The gauge transformations do not change the Newtonian frame but only the way in which the events of $M$ are labeled.

A special class of gauge transformations are those for which $\alpha=C t$ with $C$ constant. Indeed, they leave the gauge potential $b_{t}$ unchanged but change by an additive constant $\psi U$, and hence any function $\mathcal{U}$ of the form $\mathcal{U}=\psi U+K_{1} \psi+K_{2}$, $K_{1}, K_{2} \in \mathbb{R}$. Indeed we shall see that suitable constants $K_{1}$ and $K_{2}$ exist which allow us to interpret $\mathcal{U}$ (and hence $U$ for a Bargmann structure) as the classical potential. However, one should keep in mind that $U$ (and hence $\mathcal{U}$ ) is not uniquely determined because of the existence of the wider class of gauge transformations given above. Nevertheless, if the connections $\omega_{t}$ are flat a section $\sigma$ exists such that, for every $t$, $b_{t}=0$. For this special class of non rotating Newtonian frames it is possible to identify uniquely (up to an additive time function) a potential function $\mathcal{U}$.

A trivial consequence of definition 2.4 is

Lemma 2.6. The Eisenhart spacetime is a causal spacetime.

Proof. Let $V(\lambda)=\partial / \partial \lambda$ be the tangent vector to a future directed causal curve $x(\lambda)$. Since $V$ and $n$ are future directed $g(n, V) \leq 0$ or $\mathrm{d} t[V] \geq 0$ where the equality sign holds iff $V \propto n$. Thus as $t(\lambda)$ is a non-decreasing function the causal curve can not be closed unless $t(\lambda)=$ cnst. in which case $x$ is a geodesic generated by $n$ which is not closed neither.

\subsection{The $\frac{1}{\mu}$-lift.}

Given a future directed field $v(e)$ on $E$ the representative field $V$ on $M, \pi_{*} V=v$, is not uniquely defined. Nevertheless, it can be uniquely identified by imposing some conditions on its norm. We have the following

Lemma 2.7. Let $V(m)$ be a representative field on $M$ which projects on a future directed field $v(e)$ on $E$, and let $\omega(\cdot)=-g(\cdot, V)$. The following conditions are equivalent 
(i) $L_{n} V=0$,

(ii) $L_{n} \omega=0$,

(iii) $L_{n} g(V, V)=0$,

and $V(m)$ is uniquely identified if they hold and the function $g(V, V)(e)$ on $E$ is given. In particular $V(m)$ can be chosen to be causal, and for any given function $\frac{1}{\mu}: E \rightarrow[0,+\infty)$, it is uniquely identified by imposing

$$
\frac{1}{\mu}=\frac{\sqrt{-g(V, V)}}{-g(n, V)},
$$

in which case $V$ is said to be the $\frac{1}{\mu}$-lift of $v$.

Proof. Since $V$ is a representative, $L_{n} V=\beta n$ for a suitable field $\beta: M \rightarrow \mathbb{R}$, moreover $g(n, V)<0$ since $v$ is future directed. Hence

$$
\beta g(n, V)=g\left(L_{n} V, V\right)=\frac{1}{2} L_{n} g(V, V),
$$

implies that (i), i.e. $\beta=0$, iff (iii). Since $n$ is Killing (i) iff (ii). Let $V^{\prime}$ be a representative. By choosing a suitable field $\alpha: M \rightarrow \mathbb{R}$, we can find another representative $V=V^{\prime}+\alpha n$ such that

$$
g(V, V)=g\left(V^{\prime}, V^{\prime}\right)+2 g\left(V^{\prime}, n\right) \alpha=g\left(V^{\prime}, V^{\prime}\right)-2(\psi \mathrm{d} t[v]) \alpha,
$$

is any prescribed function, and a condition of this kind fixes the function $\alpha$ and hence the representative. In particular $V$ can be chosen to be causal and such that Eq. (19) holds with $-g(n, V)=\psi \mathrm{d} t[v]>0$.

In what follows we shall be interested only in the case in which $\mu$ is a constant throughout $E$. The reason for introducing the function $\mu$ stands on the fact that for future directed timelike geodesic on $M$ both $\sqrt{-g(V, V)}$ and $-g(n, V)$ are positive constants where $V$ is the tangent vector. Then $\mu$ is independent of the affine parametrization and it is the momentum conjugate to $y$ with respect to the proper time action (see section 3 ).

Note that if $\psi \mathrm{d} t[v]=1$, i.e. $v(e)$ is a Newtonian frame, then $-g(n, V)=1$. The 0 -lift corresponds to $V$ lightlike, and will be called the lightlike or light lift of $v$. The $\frac{1}{\mu}$-lift makes sense not only for vector fields but also for vectors and if $V$ is the $\frac{1}{\mu}$-lift of $v$ then $\lambda V, \lambda>0$, is the $\frac{1}{\mu}$-lift of $\lambda v$.

Let $V$ be the tangent vector to a causal curve on $M$, which projects to a future directed curve of tangent vector $v$ (i.e. $g(n, V)<0$ ). The curves on $E$ and $M$ can be parametrized with respect to $t$, and Eq. (19) reads

$$
\dot{y}=\frac{1}{2 \psi} a_{t}(\dot{q}, \dot{q})+b_{t}(\dot{q})+\psi\left(\frac{1}{2 \mu(t)^{2}}-\frac{1}{2}-U\right) .
$$

As we shall see the curves for which $1 / \mu$ is a constant will be particularly interesting. 


\subsection{Timelike Killing fields and conservation of energy}

Given a Newtonian frame $v$ and a choice of representative $V$ such that condition (i) holds, a gauge transformation can be made in such a way that $L_{V} y=0$ or, which is the same, $V=\frac{1}{\psi} \frac{\partial}{\partial t}$. Conversely, whatever the gauge chosen the field $\frac{1}{\psi} \frac{\partial}{\partial t}$ is a representative which commutes with $n=\partial / \partial y$. These expressions are kept unaltered by the restricted gauge transformations for which $\alpha$ in Eqs. (16)-(18) does not depend on $t$.

Consider a Bargmann structure $(\psi=1)$. The change of gauge $y^{\prime}=y-C t$, where $C$ is a constant, leaves unaltered the fields $a_{t}, b_{t}$, and the expression $n=\partial / \partial y$, while it changes the function $U$ and the field $\partial / \partial t$

$$
\begin{aligned}
U^{\prime} & =U+C, \\
\frac{\partial}{\partial t^{\prime}} & =\frac{\partial}{\partial t}+C \frac{\partial}{\partial y} .
\end{aligned}
$$

Notice that depending on the event and on the gauge chosen the representative $\frac{\partial}{\partial t}$ may be timelike, lightlike or spacelike. The previous observation is useful because it shows that if $\frac{\partial}{\partial t}$ is Killing then it is not restrictive to assume it timelike, provided the function $U$ is bounded from below.

Theorem 2.8. In a Bargmann structure let $V, L_{n} V=0$, be a representative field for the Newtonian frame $v$. The field $\partial / \partial t$ on $M$ is always a representative for $v$, and the section $\sigma$ can be chosen in a way such that $V=\frac{\partial}{\partial t}$ on $M$, and $v=\frac{\partial}{\partial t}$ on $E$. With that choice $V$ is Killing iff $a_{t}, b_{t}$ and $U$ are independent of $t$ (but note that if $a_{t}$ depends on then $V$ is not Killing irrespective of the choice of $\sigma$ ). Moreover, an (alternative) timelike Killing representative can be found iff the potential $U$ is bounded from below.

Proof. The first statement is trivial. The causal character of $V=\frac{\partial}{\partial t}$ is given by the sign of the function $(2 U+1)$ in Eisenhart's metric. Clearly if $U$ is bounded from below a gauge transformation $y^{\prime}=y-C t$ can be found such that, due to Eq. (21), $\left(2 U^{\prime}+1\right)>0$, i.e. $\frac{\partial}{\partial t^{\prime}}$ is timelike, and, due to Eq. (22), $\frac{\partial}{\partial t^{\prime}}$ being a linear combination of Killing fields is Killing. Every alternative Killing representative has the form $\frac{\partial}{\partial t}+C(m) n$. Since both $n$ and $\frac{\partial}{\partial t}$ are Killing, the Killing condition reads, $C_{; \mu} n_{\nu}+C_{; \nu} n_{\mu}=0$ or $\mathrm{d} C \otimes \mathrm{d} t+\mathrm{d} t \otimes \mathrm{d} C=0$ which implies that $C$ is a constant. Thus any alternative Killing representative is obtained through the already considered gauge transformation.

Consider a Bargmann structure with a (timelike) Killing field $K$ which projects to a Newtonian frame i.e. let $L_{K} g=0, L_{n} K \propto n$ and $g(K, n)=-1$. Set $L_{n} K=$ $[n, K]=\beta n$. From $0=L_{K} g(K, n)=g\left(K, L_{K} n\right)=-g\left(K, L_{n} K\right)=-\beta g(K, n)=\beta$ we conclude that $L_{n} K=0$ and hence theorem 2.8 can be applied with $V=K$. Thus the section $\sigma$ can be chosen in such a way that $K=\frac{\partial}{\partial t}$ and $a_{t}, b_{t}$ and $U$ do not depend on $t$. Consider now a future directed causal curve $x(\lambda)$ on $M$, with tangent vector $\frac{\mathrm{d} x}{\mathrm{~d} \lambda}$ nowhere proportional to $n$, i.e. $g\left(n, \frac{\mathrm{d} x}{\mathrm{~d} \lambda}\right)<0$. It is not difficult to check that

$$
\frac{1}{2} a(\dot{q}, \dot{q})+U=\frac{g\left(n, \frac{\mathrm{d} x}{\mathrm{~d} \lambda}\right) g\left(K, \frac{\mathrm{d} x}{\mathrm{~d} \lambda}\right)-\frac{1}{2} g\left(\frac{\mathrm{d} x}{\mathrm{~d} \lambda}, \frac{\mathrm{d} x}{\mathrm{~d} \lambda}\right)}{g\left(n, \frac{\mathrm{d} x}{\mathrm{~d} \lambda}\right)^{2}}-\frac{1}{2} .
$$

If $x(\lambda)$ is a causal geodesic the right-hand side is a constant of motion that does not depend on the affine parametrization. It gives the conservation of energy on the reduced spacetime $E$, and it is due to the additional symmetry generated by $K$. 


\section{Eisenhart's theorem}

We give a proof of Eisenhart's geodesic characterization of classical dynamics. A spacelike version was previously obtained using a Finslerian approach in 31] which generalizes the original theorem by Eisenhart [15. Our proof makes use of more standard tools and in particular makes use of geometrically useful constructions, such as the $\frac{1}{\mu}$-lift which allows us to find a relation between the Eisenhart's theorem in the lightlike geodesic case and a Fermat type theorem with lightlike target curves. It also allows us to apply the tools of global Lorentzian geometry to the problem of the existence of minimizers for the classical action (see theorem 5.5).

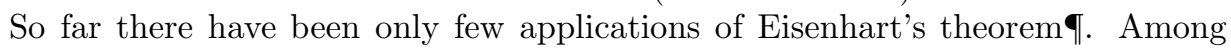
those not treated here there is one on the chaotic behavior of dynamical systems [44].

We want to find out how geodesics on spacetime $M=E \times \mathbb{R}=T \times S \times \mathbb{R}$ endowed with metric (13) project on $E$. Since $n$ is a Killing vector field $n_{\mu ; \nu}+n_{\nu ; \mu}=0$. By contraction with $n$ it follows, $n_{; \nu}^{\mu} n^{\nu}=0$ that is, the integral lines of the lightlike vector field are geodesics. We shall be interested on causal geodesics not coincident with them since these project on single points of $E$, that is they are the events of the reduced spacetime $E$. If $V \neq 0$ is a future directed causal vector not coincident with $n$ then $0>g(V, n)=-\psi \mathrm{d} t[V]$. This equation implies that the time variable $t$ increases along the projected worldlines and can be used as a time parameter.

The causal geodesics $x(\lambda)$ on which we are interested are the extremals of the action

$$
\begin{aligned}
\int_{0}^{1} \mathcal{L} \mathrm{d} \lambda^{\prime}= & \int_{0}^{1}-g\left(\frac{\mathrm{d} x}{\mathrm{~d} \lambda^{\prime}}, \frac{\mathrm{d} x}{\mathrm{~d} \lambda^{\prime}}\right) \mathrm{d} \lambda^{\prime}=\int_{0}^{1}\left\{-a_{t}\left(\frac{\mathrm{d} q}{\mathrm{~d} \lambda^{\prime}}, \frac{\mathrm{d} q}{\mathrm{~d} \lambda^{\prime}}\right)-2 \psi b_{t}\left(\frac{\mathrm{d} q}{\mathrm{~d} \lambda^{\prime}}\right) \frac{\mathrm{d} t}{\mathrm{~d} \lambda^{\prime}}\right. \\
& \left.+(2 U+1) \psi^{2}\left(\frac{\mathrm{d} t}{\mathrm{~d} \lambda^{\prime}}\right)^{2}+2 \psi \frac{\mathrm{d} t}{\mathrm{~d} \lambda^{\prime}} \frac{\mathrm{d} y}{\mathrm{~d} \lambda^{\prime}}\right\} \mathrm{d} \lambda^{\prime} .
\end{aligned}
$$

The parameter $\lambda$ is an affine parameter for the seeked extremals. There are two conserved quantities. The momentum conjugate to $y$

$$
\tilde{\mu}=2 \psi \frac{\mathrm{d} t}{\mathrm{~d} \lambda}=-2 g\left(n, \frac{\mathrm{d} x}{\mathrm{~d} \lambda}\right)>0
$$

where $\tilde{\mu}>0$ because the geodesic is causal, future directed, and not coincident with a flow line of the lightlike Killing vector field, and

$$
\begin{aligned}
\mathcal{L} & =-g\left(\frac{\mathrm{d} x}{\mathrm{~d} \lambda}, \frac{\mathrm{d} x}{\mathrm{~d} \lambda}\right)=-a_{t}\left(\frac{\mathrm{d} q}{\mathrm{~d} \lambda}, \frac{\mathrm{d} q}{\mathrm{~d} \lambda}\right) \\
& -2 \psi b_{t}\left(\frac{\mathrm{d} q}{\mathrm{~d} \lambda}\right) \frac{\mathrm{d} t}{\mathrm{~d} \lambda}+(2 U+1) \psi^{2}\left(\frac{\mathrm{d} t}{\mathrm{~d} \lambda}\right)^{2}+\tilde{\mu} \frac{\mathrm{d} y}{\mathrm{~d} \lambda}=C^{2},
\end{aligned}
$$

where $C>0$ for a timelike geodesic and $C=0$ for a lightlike geodesic. The last equation can be used in place of the Euler-Lagrange equation corresponding to the coordinate $t$. The Euler-Lagrange equations obtained from the variation with respect to $q$ can be simplified by removing $\lambda$, through Eq. (25), and $y$ through Eq. (26). The resulting equations will be called the projected Euler-Lagrange equation as the

9 Benn [4] and Szydlowski [46] cite Eisenhart [15] while studying the geodesics of a warped product spacetime with a metric of the form $g_{a b} \mathrm{~d} q^{a} \mathrm{~d} q^{b}+A\left(q^{a}\right) \mathrm{d} u^{2}$ which, according to them, leads to a Newtonian dynamics on the reduced space of coordinates $\left\{q^{a}\right\}$ by taking as time the affine parameter and by letting $A^{-1} \propto \mathcal{U}$, with $\mathcal{U}$ the Newtonian potential. However, this attribution seems incorrect. In Eisenhart's work the crossed terms of the metric are of fundamental importance, its determinant does not depend on the sign of $\mathcal{U}$, and $A \propto \mathcal{U}$ [15 p. 593 and Eq. 2.10]. Their work is more properly related to that on Kaluza-Klein theories with a scalar field. 
projection $q(t)$ on $E$ of a geodesic satisfies them. Nevertheless, we will not need to find them explicitly. Instead, our problem will be that of finding a direct variational formulation for them.

Note that the conserved quantities $\tilde{\mu}$ and $C$ depend on the chosen affine parametrization and are fixed by the requirement that at the endpoints $\lambda=0$ and $\lambda=1$, respectively. However, due to the homogeneity in $\lambda$ of the Euler-Lagrange equations, the projected Euler-Lagrange equations will be dependent only on the already introduced ratio

$$
\frac{1}{\mu}=\frac{2 C}{\tilde{\mu}}=\frac{\sqrt{-g\left(\frac{\mathrm{d} x}{\mathrm{~d} \lambda}, \frac{\mathrm{d} x}{\mathrm{~d} \lambda}\right)}}{-g\left(n, \frac{\mathrm{d} x}{\mathrm{~d} \lambda}\right)},
$$

and will be called $\frac{1}{\mu}$-Euler-Lagrange equations. Note that $\mu \in(0,+\infty]$, where $\mu=+\infty$ iff $C=0$. One can easily check that $\mu \neq 0$ is the momentum conjugate to $y$ for the Lagrangian $\mathcal{L}^{1 / 2}$ which leads to the usual proper time action (we did not start directly from that action because, in this way, we include the lightlike geodesics in our analysis).

Eq. (25) implies that in a Bargmann structure $t$ is an affine parameter for the geodesic. More generally, Eq. (26) implies that for any timelike geodesic

$$
\mathrm{d} \tau=\frac{\psi}{\mu} \mathrm{d} t
$$

where $\tau$ is the proper time. The equation (27) reads

$$
y(t)=y\left(t_{0}\right)+\int_{t_{0}}^{t}\left[\frac{1}{2 \psi} a_{t^{\prime}}(\dot{q}, \dot{q})+b_{t^{\prime}}(\dot{q})+\psi\left(\frac{1}{2 \mu^{2}}-\frac{1}{2}-U\right)\right] \mathrm{d} t^{\prime} .
$$

Definition 3.1. Given $\mu \in(0,+\infty]$, a future directed curve $(t, q(t))$ on $E$ and a point $m_{0}=\left(t_{0}, q\left(t_{0}\right), y\left(t_{0}\right)\right)$ (determined by a choice of $y_{0}=y\left(t_{0}\right)$ ) on the fiber of the starting point $e_{0}=\left(t_{0}, q\left(t_{0}\right)\right)$, equation (29) defines a new future directed causal curve on $M$, given by $(t, q(t), y(t))$, which we call the $\frac{1}{\mu}$-lift of $(t, q(t))$. The 0 -lift will also be called light lift.

The $\frac{1}{\mu}$-lift can be defined for any future directed curve on $E$ and, by construction, it is a timelike curve on $M$ if $0<\mu<+\infty$ and a lightlike curve on $M$ if $\mu=+\infty$. The final endpoint $m_{1}=\left(t_{1}, q\left(t_{1}\right), y\left(t_{1}\right)\right)$ of the $\frac{1}{\mu}$-lift lies in the fiber of the final endpoint $e_{1}$ of the original curve on $E$.

The importance of the $\frac{1}{\mu}$-lift lies in the expression for the coordinate $y$ of its second endpoint which clearly resembles the classical action.

We are generalizing here the idea of light lift introduced in 35] to the case of lightlike dimensional reduction. In 35] in order to study the motion of a relativistic particle in electromagnetic and gravitational fields, a Kaluza-Klein extension of the spacetime was considered (spacelike dimensional reduction). The additional coordinate of the light lift was proved to be related to the action of the particle, in complete analogy with the present case. The relevant difference is that here the dynamics on the base is non-relativistic while the bundle is a spacetime, while there both manifolds were relativistic spacetimes.

We have almost proved the following

Theorem 3.2. Let $(M, g), M=E \times Y=T \times S \times Y, Y=\mathbb{R}, T$ connected open subset of $\mathbb{R}$, be the spacetime endowed with the metric (13), where $a_{t}, b_{t}, U$ and $\psi$ are (all possibly time dependent) a positive definite metric, 1-form field, scalar field and positive scalar field over $S$. 
Every causal geodesic, not coincident with a flow line of $n=\partial / \partial y$, has a constant of motion $\frac{1}{\mu} \in[0,+\infty)$, and a projection to $E,(t, q(t))$, which is a $C^{2}$ stationary point of

$$
E_{1 / \mu}[q(t)]=\int_{t_{0}}^{t_{1}}\left[\frac{1}{2 \psi} a_{t}(\dot{q}, \dot{q})+b_{t}(\dot{q})-\psi\left(\frac{1}{2 \mu^{2}}+\frac{1}{2}+U\right)\right] \mathrm{d} t^{\prime},
$$

on the set $C_{e_{0}, e_{1}}^{1}$ of $C^{1}$ curves $q:\left[t_{0}, t_{1}\right] \rightarrow S$ with fixed endpoints $q\left(t_{0}\right)=q_{0}$, $q\left(t_{1}\right)=q_{1}$.

Moreover, let $\frac{1}{\mu} \in[0,+\infty)$ and let $(t, q(t))$ be a future directed curve on $E$, then $q(t)$ is a $C^{2}$ stationary point of the functional $E_{1 / \mu}$ on the set $C_{e_{0}, e_{1}}^{1}$ iff the $\frac{1}{\mu}$-lift to $M$ obtained by defining $y(t)$ as in Eq. (29) is a (unparametrized) geodesic. If $\mu \neq 0$ the geodesic is timelike and the proper time parametrization is obtained integrating $\mathrm{d} \tau=\frac{\psi}{\mu} \mathrm{d} t$. If $\frac{1}{\mu}=0$ the geodesic is lightlike and an affine parametrization is obtained integrating $\mathrm{d} \lambda=\psi \mathrm{d}$ t.

In order to complete the proof we have still to solve our main problem, i.e. to prove that the $\mu$-dependent variational principle $\delta E_{1 / \mu}[q(t)]=0$ on $E$ leads directly to the projected $\frac{1}{\mu}$-Euler-Lagrange equations and that the $\frac{1}{\mu}$-lifts of the stationary points are geodesics on $M$. In the next sections we shall complete the proof by considering separately the cases $\mu=+\infty$ and $\mu<+\infty$.

Note that not every future directed causal curve on $M$ can be regarded as a $\frac{1}{\mu}$-lift of a curve on $E$ since the function $\frac{1}{\mu}(t)$ calculated through Eq. (27) must be a constant for this to be the case. However, any lightlike curve on $M$ with tangent vectors nowhere proportional to $n$, can be regarded as the light lift of its own projection on $E$ because in this case $\frac{1}{\mu}(t)=0$.

By calculating the Euler-Lagrange equations of the functional $E_{1 / \mu}[q(t)]$ we find that the potential $b_{t}$ gives rise to a magnetic type force proportional to the curvature of the connection, that is, exactly the Coriolis force that we expected.

A consequence of the previous theorem is the following theorem which generalizes Eisenhart's.

Theorem 3.3. Let $\tilde{a}_{t}, b_{t}$ and $\mathcal{U}$ be (possibly time dependent) positive definite metric, 1-form field and scalar field (potential) on the configuration space $S$. For any choice of constant $\mu \in(0,+\infty]$, and time dependent field $\psi>0$, every $C^{2}$ stationary point $(t, q(t))$ of the classical action $\left(t_{0}<t_{1}\right)$

$$
\int_{t_{0}}^{t_{1}}\left[\frac{1}{2} \tilde{a}_{t}(\dot{q}, \dot{q})+b_{t}(\dot{q})-\mathcal{U}(t, q)\right] \mathrm{d} t^{\prime}
$$

on the set $C_{e_{0}, e_{1}}^{1}$ can be regarded, up to reparametrizations, as the projection of a causal future directed geodesic on a $(d+1)+1$-dimensional spacetime $M=T \times S \times Y$, $Y=\mathbb{R}$, endowed with the metric

$$
g_{\frac{1}{\mu}, \psi}=\psi\left[\pi_{S}^{*} \tilde{a}_{t}-\mathrm{d} t \otimes\left(\mathrm{d} y-\pi_{S}^{*} b_{t}\right)-\left(\mathrm{d} y-\pi_{S}^{*} b_{t}\right) \otimes \mathrm{d} t-\left(2 \mathcal{U}-\frac{\psi}{\mu^{2}}\right) \mathrm{d} t^{2}\right] .
$$

The geodesic is timelike if $\frac{1}{\mu} \neq 0$, in which case the proper time along the geodesic is $\mathrm{d} \tau=\frac{\psi}{\mu} \mathrm{d} t$, and lightlike if $\frac{1}{\mu}=0$, in which case an affine parametrization is $\mathrm{d} \lambda=\psi \mathrm{d} t$. Moreover, the (unparametrized) geodesic is the $\frac{1}{\mu}$-lift of the stationary point on E, i.e. it is obtained by setting

$$
y(t)=y\left(t_{0}\right)+\int_{t_{0}}^{t}\left[\frac{1}{2} \tilde{a}_{t}(\dot{q}, \dot{q})+b_{t}(\dot{q})+\left(\frac{\psi}{\mu^{2}}-\mathcal{U}\right)\right] \mathrm{d} t^{\prime} .
$$


Conversely, every future directed geodesic on $\left(M, g_{\frac{1}{\mu}, \psi}\right)$ which is, up to reparametrizations, the $\frac{1}{\mu}$-lift of its own projection $(t, q(t))$ on $E$ is such that $q(t)$ is a $C^{2}$ stationary point of the classical action on the set $C_{e_{0}, e_{1}}^{1}$. In particular, every lightlike geodesic on $\left(M, g_{0, \psi}\right)$, not coincident with a flow line of $n=\partial / \partial y$, projects on a stationary point of the classical action and every stationary point can be obtained in this way.

Recall that the stationary points of the action functional (31) do not depend on the choice of zero for the potential. Thus in Eqs. (32)-(33) $\mathcal{U}$ can be replaced with $\mathcal{U}+C$ where $C \in \mathbb{R}$ is an arbitrary constant, however note that the metric so obtained is actually isometric to the previous one since the constant can be removed with a change of coordinate $y \rightarrow y-C t$.

Theorem 3.3 follows immediately from theorem 3.2 once the identification

$$
\mathcal{U}=\psi\left(\frac{1}{\mu^{2}}+\frac{1}{2}+U\right)+\text { const. }
$$

has been made. In particular for a Bargmann structure, $\psi=1$ and $U$ is then a potential equivalent to $\mathcal{U}$.

\section{The proof}

The proof of theorem 3.2, especially in the lightlike case, is particularly interesting and will be needed for a deeped understanding of the next section.

\subsection{The lightlike geodesic case, $\mu=+\infty$}

We have to prove that the projection of a lightlike geodesic $x(\lambda)$ on $M$, not coincident with a flow line of $n$, is, once parametrized with respect to $t$, a stationary point of the functional

$$
E_{0}[q(t)]=\int_{t_{0}}^{t_{1}}\left[\frac{1}{2 \psi} a_{t}(\dot{q}, \dot{q})+b_{t}(\dot{q})-\left(U+\frac{1}{2}\right) \psi\right] \mathrm{d} t^{\prime}
$$

and conversely that the light lift of a stationary point of this functional is, with a suitable parametrization, a lightlike geodesic on $M$. In order to reach this result we recall that the unparametrized lightlike geodesics do not change under conformal changes of the metric [47, Appendix D]. As a consequence the lightlike geodesic $x(\lambda)$ on $M$ can be suitably reparametrized to become a stationary point of the action

$$
\int_{\tilde{\lambda}_{0}}^{\tilde{\lambda}_{1}} \frac{1}{2 \psi} g\left(\frac{\mathrm{d} x}{\mathrm{~d} \lambda^{\prime}}, \frac{\mathrm{d} x}{\mathrm{~d} \lambda^{\prime}}\right) \mathrm{d} \tilde{\lambda}^{\prime}=\int_{\tilde{\lambda}_{0}}^{\tilde{\lambda}_{1}}\left[\frac{1}{2 \psi} a_{t}(\dot{q}, \dot{q})+b_{t}(\dot{q})-\left(U+\frac{1}{2}\right) \psi-\dot{y}\right]\left(\frac{\mathrm{d} t}{\mathrm{~d} \tilde{\lambda}^{\prime}}\right)^{2} \mathrm{~d} \tilde{\lambda}^{\prime} .
$$

Conversely, if a causal curve $x(\tilde{\lambda})$ with tangent vectors nowhere proportional to $n$ satisfies the previous variational principle then it can be reparametrized to become a lightlike geodesic for $(M, g)$. Let $x(\tilde{\lambda}, r), r \geq 0$, be a $\left(C^{1}\right)$ variation with fixed endpoints of a causal curve $x(\tilde{\lambda})=x(\tilde{\lambda}, 0)$, with tangent vectors nowhere proportional to $n$. The continuous function in $(\tilde{\lambda}, r), g\left(n, x_{\tilde{\lambda}}(\tilde{\lambda}, r)\right)$, where $x_{\tilde{\lambda}}(\tilde{\lambda}, r)$ is the longitudinal tangent vector, is negative for $r=0$, i.e. over $x(\tilde{\lambda})$. Since the curve $x(\tilde{\lambda})$ is defined over a compact there is an $\epsilon>0$ such that for $r<\epsilon$ the longitudinal curves of the variation have tangent vectors nowhere proportional to $n$. Therefore, it is not restrictive to assume that the longitudinal curves do not have tangent vectors proportional to $n$. As a consequence the curves of the variation can be reparametrized with respect to $t$ because $\mathrm{d} t / \mathrm{d} \tilde{\lambda}=-\psi^{-1} g\left(n, x_{\tilde{\lambda}}\right)>0$. Thus there are functions $\hat{x}(t, r)$ 
and $t(\lambda, r)$ such that $x(\lambda, r)=\hat{x}(t(\lambda, r), r)$. Conversely, for any pair of functions $\hat{x}(t, r)$ and $t(\lambda, r)$ the previous equation gives a variation $x(\lambda, r)$, the only conditions to be imposed on $\hat{x}(t, r)$ and $t(\lambda, r)$ are as follows, $x(\lambda)=\hat{x}(t(\lambda, 0), 0)$ where $\hat{x}(t, 0)$ is the reparametrization with $t$ of $x(\lambda)$ and $t(\lambda, 0)$ gives the dependence between the two parametrization on the same curve, and finally, $t\left(\lambda_{0}, r\right)=t_{0}, t\left(\lambda_{1}, r\right)=t_{1}$, $\hat{x}\left(t_{0}, r\right)=x\left(\lambda_{0}\right), \hat{x}\left(t_{1}, r\right)=x\left(\lambda_{1}\right)$.

The particular form of $\hat{x}(t, r)$ and $t(\lambda, r)$ selects the variational field which reads $\left.x_{r}\right|_{r=0}=\left.\hat{x}_{t} t_{r}\right|_{r=0}+\left.\hat{x}_{r}\right|_{r=0}$. The idea is first to fix $t(\lambda, r)=t(\lambda, 0)$ and find the Euler-Lagrange equations corresponding to the freedom induced by variations of the form $x(\lambda, r)=\hat{x}(t(\lambda, 0), r)$ and then to fix $\hat{x}(t, r)=\hat{x}(t, 0)$ and find the EulerLagrange equation corresponding to the freedom induced by variations of the form $x(\lambda, r)=\hat{x}(t(\lambda, r), 0)$.

Note that $\hat{x}(t, r)=(t, q(t, r), y(t, r))$. The variation $\hat{x}(t, r)$ which keeps $q(t, r)=$ $q(t)$ but changes $y(t)$ gives the Euler-Lagrange equation

$$
\mathrm{d} t / \mathrm{d} \tilde{\lambda}=\text { cnst. }>0,
$$

that is, if $x(\lambda)$ is a lightlike geodesic for $(M, g)$ then $t$ is an affine parameter for the same curve regarded as a lightlike geodesic of $(M, g / \psi)$. The relation between $\lambda$ and $t$ is then $\mathrm{d} \lambda=K \psi \mathrm{d} t$ for a suitable constant $K \in \mathbb{R}^{+}$(see [47, Eq. (D6)]).

Using Eq. (35) it is easily shown that the variations which keep $y(t, r)=y(t)$ but change $q(t)$ lead to the same Euler-Lagrange equations of functional $E_{0}$. Indeed, the term $-\dot{y}\left(\frac{\mathrm{d} t}{\mathrm{~d} \dot{\lambda}^{\prime}}\right)^{2}$ does not change under the said variations and does not contribute to the Euler-Lagrange equations. Also the factor $\left(\frac{\mathrm{d} t}{\mathrm{~d} \tilde{\lambda}^{\prime}}\right)^{2}$ in the remaining term $\left[\frac{1}{2 \psi} a_{t}(\dot{q}, \dot{q})+b_{t}(\dot{q})-\left(U+\frac{1}{2}\right) \psi\right]\left(\frac{\mathrm{d} t}{\mathrm{~d} \tilde{\lambda}^{\prime}}\right)^{2}$ does not play any role because under integration by parts it gets differentiated with respect to $t$ and vanishes due to Eq. (35). As a result the Euler-Lagrange equations corresponding to the said variations are those of $E_{0}$.

Finally, the variation $x(\lambda, r)=\hat{x}(t(\lambda, r), 0)$ gives Eq. (29), hence every lightlike geodesic not coincident with a flow line of $n$ is the light lift of a stationary point of $E_{0}$ parametrized so that $\mathrm{d} \lambda=K \psi \mathrm{d} t$ for a suitable constant $K \in \mathbb{R}^{+}$. Conversely, the light lift of a stationary point of $E_{0}$ satisfies all the Euler-Lagrange equations of functional (34), i.e. it is a lightlike geodesic in $(M, g / \psi)$ and once parametrized with $\mathrm{d} \lambda=\psi \mathrm{d} t$ becomes a lightlike geodesic for $(M, g)$.

\subsection{The timelike geodesic case, $\mu<+\infty$}

In this case we are interested in the projection of timelike geodesics on $M$. It is convenient to consider the variational principle

$$
\delta \int_{0}^{1} \mathcal{L}^{1 / 2} \mathrm{~d} \lambda=0
$$

over the set of $C^{2}$ timelike curves with fixed endpoints. The action is the usual proper time. The cyclic variable $y$ can be removed from the variational principle using Routh's reduction [34] (see also [31] for the application to Lagrangians homogeneous of first degree in the velocities). The Routhian is a reduced Lagrangian obtained from the original Lagrangian by making a Legendre transform with respect to the velocities of the cyclic variables that have to be removed, and by considering the conserved conjugate momenta as constants. In our case we want to remove the variable $y$ and 
its velocity $\mathrm{d} y / \mathrm{d} \lambda$ so as to obtain a variational principle in the quotient space $E$. The conjugate momentum is

$$
\begin{aligned}
\mu & =\mathcal{L}^{-1 / 2} \psi \frac{\mathrm{d} t}{\mathrm{~d} \lambda} \\
& =\frac{\psi}{\left\{-a_{t}(\dot{q}, \dot{q})-2 \psi b_{t}(\dot{q})+(2 U+1) \psi^{2}+2 \psi \dot{y}\right\}^{1 / 2}},
\end{aligned}
$$

from which we obtain that $y(t)$ is given by Eq. (29). The Routhian is

$$
\begin{aligned}
R & =\mu \frac{\mathrm{d} y}{\mathrm{~d} \lambda}-\mathcal{L}^{1 / 2}=\left[\mu \dot{y}-\frac{\psi}{\mu}\right] \frac{\mathrm{d} t}{\mathrm{~d} \lambda} \\
& =\left[\frac{1}{2 \psi} a_{t}(\dot{q}, \dot{q})+b_{t}(\dot{q})-\psi\left(\frac{1}{2 \mu^{2}}+\frac{1}{2}+U\right)\right] \mu \frac{\mathrm{d} t}{\mathrm{~d} \lambda},
\end{aligned}
$$

and, up to a constant factor, the reduced action becomes

$$
\frac{1}{\mu} \int_{0}^{1} R \mathrm{~d} \lambda=\int_{t_{0}}^{t_{1}}\left[\frac{1}{2 \psi} a_{t}(\dot{q}, \dot{q})+b_{t}(\dot{q})-\psi\left(\frac{1}{2 \mu^{2}}+\frac{1}{2}+U\right)\right] \mathrm{d} t^{\prime} .
$$

The proof of theorem 3.2 and hence of theorem 3.3 are complete.

\section{The geometric interpretation of the lightlike geodesic case: Fermat's principle and Bolza's problem}

In this section $(M, g)$ is the Eisenhart spacetime $M=T \times S \times \mathbb{R}, T$ open connected subset of $\mathbb{R}$, with $g$ given by Eq. (13) with (all possibly time dependent) $a_{t}$, a positive definite metric, $b_{t}$, a 1-form field, $U$, a scalar field and $\psi>0$, a positive scalar field.

Let us consider points $e_{0}, e_{1} \in E=T \times S$ with $e_{1}$ in the future of $e_{0}, t_{1}>t_{0}$. Let $m_{0}=\left(e_{0}, y_{0}\right)$ be an event on the fiber of $e_{0}$ in the bundle $\pi: M \rightarrow E$. Consider the set $\mathcal{N}_{m_{0}, e_{1}}$ of the (unuparametrized) $C^{1}$ lightlike curves connecting $m_{0}$ to $e_{1}$ 's fiber. The coordinate $y_{1}$ of the final endpoint $m_{1}=\left(e_{1}, y_{1}\right)$ can be regarded as a functional on $\mathcal{N}_{m_{0}, e_{1}}$. Note that the parametrization $\lambda$ of the curves considered will play no role in the discussion and can always be taken such that $\lambda \in[0,1]$. Note also that the definition of stationary point for the functional $y_{1}$ is not affected by changes of the section $\sigma$, as this implies only the addition of a constant.

Lemma 5.1. If $g\left(n, \partial_{\lambda}\right)=0$ at a point of a curve $x \in \mathcal{N}_{m_{0}, e_{1}}$ then a lightlike variation $x(\lambda, r), r \geq 0$, with longitudinal curves belonging to $\mathcal{N}_{m_{0}, e_{1}}$, can be constructed such that $\mathrm{d} y_{1} / \mathrm{d} r>0$. In particular, a stationary point $x \in \mathcal{N}_{m_{0}, e_{1}}$, of the arrival coordinate $y_{1}$ on $\mathcal{N}_{m_{0}, e_{1}}$ satisfies $g\left(n, \partial_{\lambda}\right) \neq 0$ at every point. The same inequality holds for any lightlike geodesic in $\mathcal{N}_{m_{0}, e_{1}}$.

Proof. That the statement holds for any geodesic follows from the fact that $g\left(n, \partial_{\lambda}\right)(\bar{\lambda})=0, \bar{\lambda} \in[0,1]$, would imply that the lightlike geodesic $x(\lambda)$ is tangent to $n$ at a point and hence coincides with the lightlike geodesic generated by $n$, which is impossible as the initial and final endpoints of $x(\lambda)$ project on distinct points of $E$.

Now, consider a curve $x(\lambda) \in \mathcal{N}_{m_{0}, e_{1}}$. The idea is that if $g\left(n, \partial_{\lambda}\right)(\bar{\lambda})=0$, $\bar{\lambda} \in(0,1]$, then there is a lightlike variation of $x(\lambda), \lambda \in[0, \bar{\lambda}]$, which keeps the projection of the endpoint $\bar{e}=e(\bar{\lambda})$ fixed but changes the value of $\bar{y}=y(\bar{\lambda})$ linearly in the variational parameter. Then the variation of the whole curve $x(\lambda)$ is obtained by gluing the said variation up to $\bar{\lambda}$ to the Lie translation of $x(\lambda)$ along the fibers for $\lambda>\bar{\lambda}$. 
A similar idea is followed if $\bar{\lambda}=0$. In this case it suffices to follow the geodesic generated by $n$ at $m_{0}$ and then to move on the Lie translation of $x(\lambda)$ along the fiber.

If $g\left(n, \partial_{\lambda}\right)(\bar{\lambda})=0, \bar{\lambda} \in(0,1]$, we want to show that there is a lightlike variation of $x(\lambda), \lambda \in[0, \bar{\lambda}]$, which keeps the projection of the endpoint $\bar{e}=e(\bar{\lambda})$ fixed but changes the value of $\bar{y}=y(\bar{\lambda})$. To this end, let $\eta\left(y^{\prime}\right)$ be the integral line of the geodesic generated by $n$ and such that $\eta(0)=x(\bar{\lambda})$, i.e. $y^{\prime}=y-\bar{y}$, and let $k$ be the constant such that $\frac{\mathrm{d} x}{\mathrm{~d} \lambda}(\bar{\lambda})=k n$. Consider the following variation $x(\lambda, r)$ for $r \geq 0$

$$
\begin{aligned}
& x(\lambda, r)=x((1+r) \lambda), \quad 0 \leq \lambda \leq \frac{\bar{\lambda}}{1+r} \\
& x(\lambda, r)=\eta(k((1+r) \lambda-\bar{\lambda})), \quad \frac{\bar{\lambda}}{1+r} \leq \lambda \leq \bar{\lambda}
\end{aligned}
$$

it consists in extending the lightlike curve along the geodesic and in rescaling the parameter. Clearly the final endpoint being $\eta(k r \bar{\lambda})$ has a coordinate $y$ of value $\bar{y}+k \bar{\lambda} r$, which increases linearly with $r$.

Lemma 5.2. The lightlike geodesics connecting $m_{0}=\left(e_{0}, t_{0}\right)$ to $e_{1}$ 's fiber, $t_{0}<t_{1}$, are stationary points for the arrival coordinate functional $y_{1}$ on the set $\mathcal{N}_{m_{0}, e_{1}}$.

Proof. Let $x(\lambda, r)$, be a variation of $x(\lambda, 0)=x(\lambda)$ made of curves belonging to $\mathcal{N}_{m_{0}, e_{1}}$. The variational field $\partial_{r}$ vanishes at $\lambda=0$ while $\left.\partial_{r}\right|_{(\lambda, r)=(1,0)}=\frac{\mathrm{d} y}{\mathrm{~d} r} n$, hence the functional given by the function $y$ on the final endpoint has a stationary point at $x(\lambda, 0)$ iff the variational field vanishes at the final endpoint. Since $g\left(\partial_{\lambda}, \partial_{\lambda}\right)=0$,

$$
0=\partial_{r} g\left(\partial_{\lambda}, \partial_{\lambda}\right)=2 \partial_{\lambda} g\left(\partial_{\lambda}, \partial_{r}\right)-2 g\left(\nabla_{\partial_{\lambda}} \partial_{\lambda}, \partial_{r}\right),
$$

If $x(\lambda)$ is a geodesic, up to reparametrizations, $\nabla_{\partial_{\lambda}} \partial_{\lambda}=f \partial_{\lambda}$ for a certain function $f(\lambda)$. Thus $\left.g\left(\partial_{\lambda}, \partial_{r}\right)\right|_{r=0}=C \exp \left\{\int_{0}^{\lambda} f \mathrm{~d} \lambda^{\prime}\right\}$, and since $\left.\partial_{r}\right|_{\lambda=0}=0$, we have $C=0$. Finally,

$$
0=\left.g\left(\partial_{\lambda}, \partial_{r}\right)\right|_{(\lambda, r)=(1,0)}=\left.\frac{\mathrm{d} y}{\mathrm{~d} r} g\left(\partial_{\lambda}, n\right)\right|_{(\lambda, r)=(1,0)} .
$$

But $\left.g\left(\partial_{\lambda}, n\right)\right|_{(\lambda, s)=(1,0)} \neq 0$ by lemma 5.1, hence if $x(\lambda, 0)$ is a lightlike geodesic then it is a stationary point for the functional $y_{1}$.

Consider the set $\tilde{\mathcal{N}}_{m_{0}, e_{1}} \subset \mathcal{N}_{m_{0}, e_{1}}$, made of those curves with tangent vectors newhere proportional to $n$. By lemma 5.1 the stationary points for functional $y_{1}$, and the geodesics on $\mathcal{N}_{m_{0}, e_{1}}$ belong in fact to $\tilde{\mathcal{N}}_{m_{0}, e_{1}}$. Note also that if $x(\lambda)$ is a stationary point for variations having longitudinal curves in $\mathcal{N}_{m_{0}, e_{1}}$ then the same is true for variations restricted to $\tilde{\mathcal{N}}_{m_{0}, e_{1}}$.

Let $(t, q(t))$ be a $\left(C^{1}\right)$ future directed curve connecting $e_{0}$ to $e_{1}$ and let $x(\lambda)$, $\lambda \in[0,1]$, be its light lift of starting event $m_{0}=\left(e_{0}, y_{0}\right)$ and final endpoint $m_{1}=\left(e_{1}, y_{1}\right)$ on $e_{1}$ 's fiber. Here the parametrization $\lambda$ is not important as long as $\mathrm{d} t / \mathrm{d} \lambda>0$.

The light lift belongs to $\tilde{\mathcal{N}}_{m_{0}, e_{1}}$ and every curve in $\tilde{\mathcal{N}}_{m_{0}, e_{1}}$ is the light lift of a future directed connecting curve on $E$, where $y_{1}$ is given by Eq. (29) with $\frac{1}{\mu}=0$. As a consequence we have proved again the 'if' part of 
Theorem 5.3. Let $a_{t}, b_{t}, U$ and $\psi>0$ be (possibly time dependent) positive definite metric, 1-form field and scalar fields on the d-dimensional configuration space $S$. The (classical) action functional

$$
\left\{y\left(t_{1}\right)-y\left(t_{0}\right)\right\}[q(t)]=\int_{t_{0}}^{t_{1}}\left[\frac{1}{2 \psi} a_{t}(\dot{q}, \dot{q})+b_{t}(\dot{q})-\left(U+\frac{1}{2}\right) \psi\right] \mathrm{d} t^{\prime},
$$

is stationary on $q(t)$ if and only if the light lift $(t, q(t), y(t))$ on $M$ endowed with the metric (13) is a (unparametrized) lightlike geodesic. In particular the 0-Euler-Lagrange equations are the Euler-Lagrange equations of this functional.

The 'only if' part has been proved through the argument of subsection 4.1 indeed we can use the fact that for $\frac{1}{\mu}=0$ the expressions for functional $E_{0}$ and $y_{1}$ differ only by a constant (compare Eq. (30) with Eq. (29)). Incidentally, by proving the 'only if' part we showed that the stationary point of the functional $y_{1}$ on the set $\tilde{\mathcal{N}}_{m_{0}, e_{1}}$ is a geodesic and hence, by lemma 5.2. that it is also a stationary point with respect to variations in the larger space of longitudinal curves $\mathcal{N}_{m_{0}, e_{1}}$. Summarizing we proved that, given the functional $y_{1}$, any stationary point on the set $\mathcal{N}_{m_{0}, e_{1}}$ belongs to $\tilde{\mathcal{N}}_{m_{0}, e_{1}}$, and it is a stationary point for the restricted variations belonging to $\tilde{\mathcal{N}}_{m_{0}, e_{1}}$ (lemma 5.1). These last stationary points are indeed geodesics (theorem [5.3), and hence belong to $\mathcal{N}_{m_{0}, e_{1}}$ and are stationary points with respect to the enlarged set of variations (lemma 5.2). The conclusion is that

Theorem 5.4. The stationary points of the functional $y_{1}$ over the set $\mathcal{N}_{m_{0}, e_{1}}$ of lightlike $C^{1}$ curves connecting $m_{0}=\left(e_{0}, y_{0}\right)$ to $e_{1}$ 's fiber, $t_{0}<t_{1}$, are the lightlike geodesics belonging to $\mathcal{N}_{m_{0}, e_{1}}$.

This is a modified Fermat type theorem in which the target curve is not timelike but lightlike. It is likely that the theorem could be generalized to arbitrary spacetimes, not necessarily with a lightlike Killing vector field, as it has been proved in the timelike case $[27,43]$.

\subsection{The Bolza problem}

The Lagrange problem, sometimes referred to as the Bolza problem, 7, 23, 10, 16, 8, asks to determine under which conditions the action functional has a stationary point and in particular whether it has a minimum. A classical result is Tonelli's theorem [10, 33. which states that a minimum exists provided $a_{t}$ is independent of time, $b_{t}=0$, $(S, a)$ is a complete manifold, and the Lagrangian $L$ is superlinear, that is, for all $A \in \mathbb{R}$ there is a $B \in \mathbb{R}$ such that $L(q, \dot{q}, t) \geq A \sqrt{a(\dot{q}, \dot{q})}-B$ for all $t, q, \dot{q}$. Unfortunately, these requirements are too restrictive, they imply for instance that the potential is bounded from above. For this reason other theorems have been given in the literature which replace superlinearity with other suitable growth conditions [16, 20, 8, 9 , on $U$ or $\nabla U$.

A more general approach is followed here where these growth conditions are regarded as accessory conditions needed to prove the causal simplicity 38, of the spacetime $(M, g)$ under which the existence of a minimum for the classical action follows. Indeed, remarkably, we are able to prove the following theorem which reduces the Bolza problem for the classical action to an often simpler problem in global Lorentzian geometry. It also summarizes some relevant results obtained in the lightlike geodesic case $\frac{1}{\mu}=0$. 


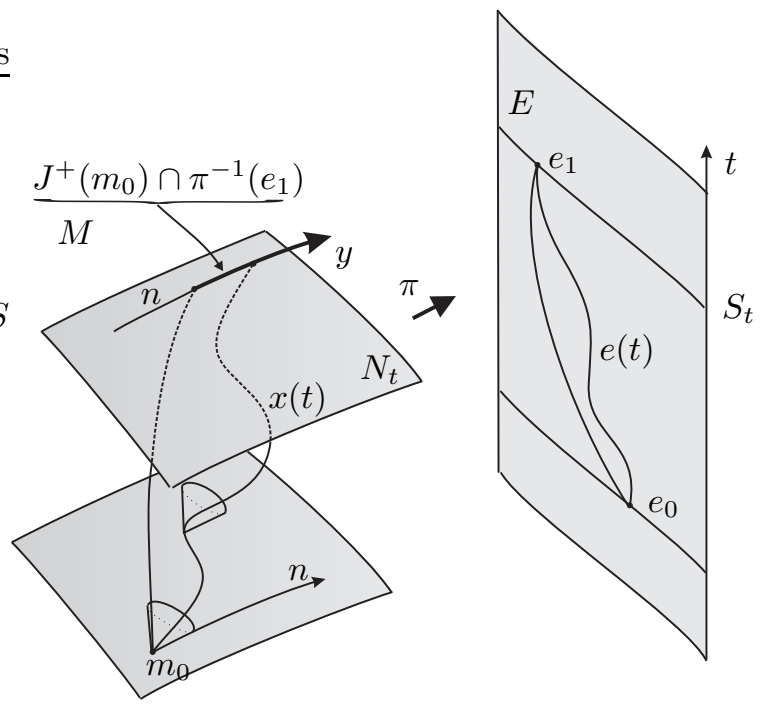

Figure 2. Every future directed curve $e(t)=(t, q(t))$ connecting two events $e_{0}$ and $e_{1}$ in $E$ is the projection of its light lift $x(t)$ i.e. a lightlike, and hence causal, curve on $M$ which starts from a given event $m_{0}$ on $e_{0}$ 's fiber and ends on $e_{1}$ 's fiber. The coordinate $y$ on the light lift second endpoint equals, up to an additive constant, the classical action functional 38. By a generalized Fermat's theorem the lightlike geodesics are the stationary points of this modified 'time'of-arrival functional, for variations restricted to lightlike curves (see theorem 5.4). Moreover, if the action (38) is bounded from below and the spacetime $(M, g)$ is causally simple then there is a lightlike geodesic that reaches a minimum for the coordinate $y$, and hence its projection is an absolute minimum of the classical action (see theorem 5.5).

Theorem 5.5. Let $S$ be a d-dimensional manifold endowed with the (all possibly time dependent) positive definite metric $a_{t}$, 1-form field $b_{t}$ and potential function $\mathcal{U}$ (all $\left.C^{r}, r \geq 1\right)$. On the classical spacetime $E=T \times S$ let $t$ be the time coordinate and let $e_{0}=\left(t_{0}, q_{0}\right)$ and $e_{1}=\left(t_{1}, q_{1}\right)$ be events, the latter in the future of the former i.e. $t_{1}>t_{0}$. Consider the classical action functional

$$
I_{e_{0}, e_{1}}[q]=\int_{t_{0}}^{t_{1}}\left[\frac{1}{2} a_{t}(\dot{q}, \dot{q})+b_{t}(\dot{q})-\mathcal{U}(t, q)\right] \mathrm{d} t
$$

on the space $C_{e_{0}, e_{1}}^{1}$ of $C^{1}$ curves $q:\left[t_{0}, t_{1}\right] \rightarrow S$ with fixed endpoints $q\left(t_{0}\right)=q_{0}$, $q\left(t_{1}\right)=q_{1}$. Construct the spacetime $M=E \times Y=T \times S \times Y, Y=\mathbb{R}$, and denote with $\pi_{S}: M \rightarrow S$, the canonical projection. Assign to $M$ the Lorentzian metric

$$
g=\pi_{S}^{*} a_{t}-\mathrm{d} t \otimes\left(\mathrm{d} y-\pi_{S}^{*} b_{t}\right)-\left(\mathrm{d} y-\pi_{S}^{*} b_{t}\right) \otimes \mathrm{d} t-2 \mathcal{U} \mathrm{d} t^{2} .
$$

(a) Every lightlike geodesic of $(M, g)$ not coincident with a flow line of $n=\partial / \partial y$, admits as affine parameter the function $t$, and for any such curve $x(t)=$ $(t, q(t), y(t))$, the function $q(t)$ is a $C^{2}$ stationary point of functional (38) on $C_{e_{0}, e_{1}}^{1}$ and $x(t)$ is the light lift of $e(t)=(t, q(t))$, that is

$$
y(t)=y(0)+I_{e_{0}, e(t)}\left[\left.q\right|_{[0, t]}\right] .
$$

Conversely, given a $C^{2}$ stationary point $q(t)$ of functional (38) on $C_{e_{0}, e_{1}}^{1}$, the light lift $x(t)=\left(t, q(t), y(0)+I_{e_{0}, e(t)}\left[\left.q\right|_{[0, t]}\right]\right)$ is an affinely parametrized lightlike geodesic of $(M, g)$ necessarily not coincident with a flow line of $n$. 
(b) The spacetime $(M, g)$ is causally simple if and only if the following two properties hold

(i) The function $\mathcal{I}: E \times E \rightarrow[-\infty,+\infty]$ given by

$$
\begin{array}{ll}
\mathcal{I}\left(e_{0}, e_{1}\right)=\inf _{q \in C_{e_{0}, e_{1}}^{1} I_{e_{0}, e_{1}}[q],} & \text { for } t_{0}<t_{1}, \\
\mathcal{I}\left(e_{0}, e_{1}\right)=+\infty, & \text { for }\left(t_{0}=t_{1} \text { and } q_{0} \neq q_{1}\right) \text { or } t_{0}>t_{1}, \\
\mathcal{I}\left(e_{0}, e_{1}\right)=0, & \text { for } t_{0}=t_{1} \text { and } q_{0}=q_{1},
\end{array}
$$

is lower semi-continuous.

(ii) If $\mathcal{I}\left(e_{0}, e_{1}\right), t_{0}<t_{1}$, is finite then the functional $I_{e_{0}, e_{1}}[q]$ attains its minimum at a certain (not necessarily unique) $\bar{q}(t) \in C_{e_{0}, e_{1}}^{1}$, i.e. $I_{e_{0}, e_{1}}[\bar{q}]=\mathcal{I}\left(e_{0}, e_{1}\right)$.

(c) Whether or not $(M, g)$ is causally simple, each minimizer $\bar{q}(t) \in C_{e_{0}, e_{1}}^{1}$, is not only $C^{1}$ but also $C^{r+1}$ differentiable and hence satisfies the Euler-Lagrange equations of $I_{e_{0}, e_{1}}$.

Proof. The statement (a) is nothing but theorem 3.3 for $\frac{1}{\mu}=0$ and $\psi=1$. Thus we have only to prove (b).

Let $\mathcal{C}_{m_{0}, e_{1}}$ be the space of $C^{1}$ causal curves connecting $m_{0}$ to $e_{1}$ 's fiber, with $t_{0}<t_{1}$ (see figure 22). Because, of the translational invariance $m_{0}$ can be arbitrarily chosen. Consider a causal curve $x(\lambda) \in \mathcal{C}_{m_{0}, e_{1}}, \lambda \in[0,1]$, which is timelike at some point. By [21, Prop. 4.5.10] there is a timelike curve $\tilde{x}(\lambda)$ with the same endpoints $m_{0}$ and $m_{1}=\left(e_{1}, y_{1}[x(\lambda)]\right)$. It is easy to prove using Eq. (20) that, since $\frac{1}{\mu}>0$ all over $\tilde{x}(\lambda)$, the coordinate $y$ evaluated at its final endpoint (which coincides with the final endpoint of $x(\lambda)$ ) is higher than the same coordinate evaluated at the final endpoint of the light lift of the projection $\tilde{e}(\lambda)$ of $\tilde{x}(\lambda)$.

Thus for every connecting curve which stays in $\mathcal{C}_{m_{0}, e_{1}}$, there is another in $\mathcal{N}_{m_{0}, e_{1}} \subset \mathcal{C}_{m_{0}, e_{1}}$ with an equal or lower value of the functional $y_{1}$. Thus

$$
\inf _{x \in \mathcal{N}_{m_{0}, e_{1}}} y_{1}[x]=\inf _{x \in \mathcal{C}_{m_{0}, e_{1}}} y_{1}[x] .
$$

Consider now a curve $x \in \mathcal{N}_{m_{0}, e_{1}}$, such that $g(n, \mathrm{~d} x / \mathrm{d} \lambda)=0$ at some point. It can not be $g(n, \mathrm{~d} x / \mathrm{d} \lambda)=0$ everywhere otherwise $x$ would coincide with a segment of geodesic generated by $n$, and its projection would not connect two different points $e_{0}$ and $e_{1}$. Thus it is not a lightlike geodesic and by [21, Prop. 4.5.10] there is a timelike curve $\hat{x}$ with the same endpoints $m_{0}$ and $m_{1}=\left(e_{1}, y_{1}[x(\lambda)]\right)$, and finally the light lift of the projection $\hat{e}$ gives a lightlike curve with a lower value of the coordinate $y_{1}$ of the second enpoint. Thus for every connecting curve which stays in $\mathcal{N}_{m_{0}, e_{1}}$, there is another in $\tilde{\mathcal{N}}_{m_{0}, e_{1}} \subset \mathcal{N}_{m_{0}, e_{1}}$ with an equal or lower value of the functional $y_{1}$. Thus

$$
\inf _{x \in \mathcal{N}_{m_{0}, e_{1}}} y_{1}[x]=\inf _{x \in \tilde{\mathcal{N}}_{m_{0}, e_{1}}} y_{1}[x] .
$$

But every curve in $\tilde{\mathcal{N}}_{m_{0}, e_{1}}$ is the light lift of its projection i.e. of some curve $e(t)=(t, q(t)), q \in C_{e_{0}, e_{1}}^{1}$. As a consequence,

$$
\inf _{x \in \tilde{\mathcal{N}}_{m_{0}, e_{1}}} y_{1}[x]=y_{0}+\inf _{q \in C_{e_{0}, e_{1}}^{1}} I_{e_{0}, e_{1}}[q],
$$

and finally,

$$
\inf _{x \in \mathcal{C}_{m_{0}, e_{1}}} y_{1}[x]=y_{0}+\inf _{q \in C_{e_{0}, e_{1}}^{1}, e_{1}} I_{e_{0}, e_{1}}[q]=y_{0}+\mathcal{I}\left(e_{0}, e_{1}\right) .
$$


We are ready to prove (c). Since $I^{+}$is open every $C^{1}$ absolute minimum $\bar{q}$, must have a light lift which connects $m_{0}$ to a point in $\dot{J}^{+}\left(m_{0}\right)=E^{+}\left(m_{0}\right)$, and hence the light lift is a maximal lightlike geodesic. The geodesic equation depends on the first derivatives of the given fields $b_{t}, a_{t}, \mathcal{U}$, and on the second derivative of the coordinates. Hence the geodesic has second derivatives which are $C^{r-1}$ differentiable, and the projection $\bar{q}(t)$ of the geodesic is $C^{r+1}$ differentiable.

Let us assume $(M, g)$ is causally simple and prove property (ii). If the action $I_{e_{0}, e_{1}}[q(t)]$ is bounded from below then there is an event on $e_{1}$ 's fiber,

$$
\bar{m}_{1}=\left(e_{1}, \bar{y}_{1}\right)=\left(e_{1}, y_{0}+\inf _{q \in C_{e_{0}, e_{1}}^{1}} I_{e_{0}, e_{1}}[q]\right),
$$

which belongs to $\dot{J}^{+}\left(m_{0}\right)$, the boundary of the causal future of $m_{0}$. If $(M, g)$ is causally simple, as it is well known [21], $\dot{J}^{+}\left(m_{0}\right)=E^{+}\left(m_{0}\right)$, that is, there is a (maximal but not necessarily unique) lightlike geodesic $\bar{x}$ connecting $m_{0}$ and $\bar{m}_{1}$. Since it is lightlike, it is the light lift of its own projection $(t, \bar{q}(t))$, and therefore, $\bar{q}(t)$ is an absolute minimum for the classical action.

Let us assume that $(M, g)$ is causally simple and prove property (i). We can assume that $\mathcal{I}\left(e_{0}, e_{1}\right) \neq-\infty$ otherwise the statement is trivial at $\left(e_{0}, e_{1}\right)$.

Let $t_{0}<t_{1}$ and assume by contradiction that $\mathcal{I}$ is not lower semi-continuous at $\left(e_{0}, e_{1}\right) \in E \times E$, then there is an $\epsilon>0$ and a sequence $\left(e_{0 k}, e_{1 k}\right) \rightarrow\left(e_{0}, e_{1}\right)$ such that $\mathcal{I}\left(e_{0 k}, e_{1 k}\right)<\mathcal{I}\left(e_{0}, e_{1}\right)-\epsilon$ (note that for $t_{0}<t_{1}, \mathcal{I}\left(e_{0}, e_{1}\right)$ is bounded from above). Without loss of generality we can assume $t_{0 k}<t_{1 k}$.

Fixed $y_{0} \in \mathbb{R}$, set $m_{0 k}=\left(e_{0 k}, y_{0}\right), m_{0}=\left(e_{0}, y_{0}\right)$, so that $m_{0 k} \rightarrow m_{0}$. We have shown that for any $q \in C_{e_{0 k}, e_{1 k}}^{1}$, the event

$$
\left(e_{1 k}, y_{0}+I_{e_{0 k}, e_{1 k}}[q]\right)
$$

belongs to $J^{+}\left(m_{0 k}\right)$ and hence the same holds for

$$
\left(e_{1 k}, y_{0}+I_{e_{0 k}, e_{1 k}}[q]+C\right)
$$

where $C$ is an arbitrary positive constant (simply reach the previous point and move along the geodesic generated by $n$ in the forward direction). For $k$ sufficiently large there is a $q \in C_{e_{0 k}, e_{1 k}}^{1}$ such that $I_{e_{0 k}, e_{1 k}}[q]<\mathcal{I}\left(e_{0}, e_{1}\right)-\epsilon$. Finally, defined $y_{1 k}$ and $y_{1}$, as $y_{1 k}=y_{1}=y_{0}+\mathcal{I}\left(e_{0}, e_{1}\right)-\frac{\epsilon}{2}$, we obtain that

$$
m_{1 k}=\left(e_{1 k}, y_{1 k}\right)=\left(e_{1 k}, y_{0}+\mathcal{I}\left(e_{0}, e_{1}\right)-\frac{\epsilon}{2}\right)
$$

belongs to $J^{+}\left(m_{0 k}\right)$. But $m_{1 k} \rightarrow m_{1}=\left(e_{1}, y_{1}\right)=\left(e_{1}, y_{0}+\mathcal{I}\left(e_{0}, e_{1}\right)-\frac{\epsilon}{2}\right)$ and by causal simplicity $m_{1} \in J^{+}\left(m_{0}\right)$ which due to Eq. (40) it is impossible.

An analogous argument works for $t_{0}=t_{1}$. Here we have to consider two possibilities, $q_{0} \neq q_{1}$ and $q_{0}=q_{1}$.

In the former case if $\mathcal{I}$ were not lower semi-continuous at $\left(e_{0}, e_{1}\right) \in E \times E$, then there would be an $M>0$ and a sequence $\left(e_{0 k}, e_{1 k}\right) \rightarrow\left(e_{0}, e_{1}\right)$ such that $\mathcal{I}\left(e_{0 k}, e_{1 k}\right)<M$. Without loss of generality we can assume $q_{0 k} \neq q_{1 k}$ and hence necessarily $t_{0 k}<t_{1 k}$ (otherwise $\mathcal{I}\left(e_{0 k}, e_{1 k}\right)=+\infty$ ). Fixed $y_{0} \in \mathbb{R}$ and arguing as above it follows that, $m_{1 k}=\left(e_{1 k}, y_{0}+M\right) \in J^{+}\left(m_{0 k}\right)$, where $m_{0 k}=\left(e_{0 k}, y_{0}\right)$. By causal simplicity $m_{1}=\left(e_{1}, y_{0}+M\right) \in J^{+}\left(m_{0}\right)$, where $m_{0}=\left(e_{0}, y_{0}\right)$ which is impossible because no causal curve can connect two events in the same slice $t=$ cnst. unless both events lie in the same geodesic generated by $n$ in which case $q_{0}=q_{1}$, which is excluded by assumption. 
In the latter case $e_{1}=e_{0}$ and if $\mathcal{I}$ were not lower semi-continuous at $\left(e_{0}, e_{1}\right) \in$ $E \times E$, then there would be and $\epsilon>0$ and a sequence $\left(e_{0 k}, e_{1 k}\right) \rightarrow\left(e_{0}, e_{1}\right)$ such that $\mathcal{I}\left(e_{0 k}, e_{1 k}\right)<\mathcal{I}\left(e_{0}, e_{1}\right)-\epsilon=-\epsilon$. The inequality implies that necessarily $t_{0 k}<t_{1 k}$, and proceeding as above we obtain that $m_{1}=\left(e_{1}, y_{0}-\frac{\epsilon}{2}\right)=\left(e_{0}, y_{0}-\frac{\epsilon}{2}\right) \in J^{+}\left(m_{0}\right)$, $m_{0}=\left(e_{0}, y_{0}\right)$, which is impossible because the only causal curve which connects $m_{0}$ to $m_{1}$ is a past directed null geodesic generated by $n$. The lower semi-continuity for $t_{1}<t_{0}$ is obvious because this is an open subset of $E \times E$ where $\mathcal{I}$ has constant value $+\infty$.

Now, assume that properties (i) and (ii) hold and let us prove that $(M, g)$ is causally simple. Since the Eisenhart spacetime is causal (lemma 2.6) we have only to prove that for every $m \in M, J^{+}(m)$ is closed [38, Def. 3.63] 6] (the analogous past case property can be proved similarly). Otherwise there are two events $m_{0}=\left(e_{0}, y_{0}\right)$ and $m_{1}=\left(e_{1}, y_{1}\right)$ such that $m_{1} \in \bar{J}^{+}\left(m_{0}\right)$ but $m_{1} \notin J^{+}\left(m_{0}\right)$.

If $t_{0}<t_{1}$, by the same argument used above, for any $q \in C_{e_{0}, e_{1}}^{1}$ and $C \geq 0$

$$
\left(e_{1}, y_{0}+I_{e_{0}, e_{1}}[q]+C\right)
$$

belongs to $J^{+}\left(m_{0}\right)$. Thus, if $\mathcal{I}\left(e_{0}, e_{1}\right)=-\infty, m_{1} \in J^{+}\left(m_{0}\right)$ the searched contradiction. Let $\mathcal{I}\left(e_{0}, e_{1}\right)$ be finite, then by property (ii) there is a minimizing curve $(t, \bar{q}(t))$ whose light lift starting at $m_{0}$ ends at $\bar{m}_{1}=\left(e_{1}, \bar{y}_{1}\right)=\left(e_{1}, y_{0}+\mathcal{I}\left(e_{0}, e_{1}\right)\right)$. Moreover, if $\bar{y}_{1} \leq y_{1}$ then $m_{1} \in J^{+}\left(m_{0}\right)$ (simply move from $m_{0}$ to $\bar{m}_{1}$ through the light lift of $(t, \bar{q}(t))$ and then along the fiber passing through $m_{1}$ in the forward direction) a contradiction. We conclude that $y_{1}<\bar{y}_{1}$. We are going to show that this fact is incompatible with property (i). Indeed, let $m_{1 k}=\left(e_{1 k}, y_{1 k}\right) \in J^{+}\left(m_{0}\right)$ be a sequence such that $m_{1 k} \rightarrow m_{1}$. There is an $\epsilon>0$ such that for sufficiently large $k, y_{1 k}<\overline{y_{1}}-\epsilon$ then $\mathcal{I}\left(e_{0}, e_{1 k}\right)<\mathcal{I}\left(e_{0}, e_{1}\right)-\epsilon$ which contradicts the lower semi-continuity of $\mathcal{I}$.

If $t_{0}=t_{1}$ and $q_{0} \neq q_{1}$, it is $\mathcal{I}\left(e_{0}, e_{1}\right)=+\infty$. Let $m_{1 k}=\left(e_{1 k}, y_{1 k}\right) \in J^{+}\left(m_{0}\right)$ be a sequence such that $m_{1 k}=\left(e_{1 k}, y_{1 k}\right) \rightarrow m_{1}=\left(e_{1}, y_{1}\right)$. We can assume without loss of generality $q_{1 k} \neq q_{0}$, and hence $t_{1 k}>t_{0}$ (because all the events of $J^{+}\left(m_{0}\right)$ have time greater than $t_{0}$ unless they lie in the geodesic generated by $n$ which pass through $m_{0}$ in which case they would project on $\left.\left(q_{0}, t_{0}\right)\right)$. Since $y_{1 k} \rightarrow y_{1}$, there is a $M>0$ such that $y_{1 k}<M$ thus $\mathcal{I}\left(e_{0}, e_{1 k}\right)<M$, in contradiction with the lower semi-continuity of $\mathcal{I}$.

If $t_{0}=t_{1}$ and $q_{0}=q_{1}$, it is $\mathcal{I}\left(e_{0}, e_{1}\right)=0$. Let $m_{1 k}=\left(e_{1 k}, y_{1 k}\right) \in J^{+}\left(m_{0}\right)$ (in particular $t_{1 k}>t_{0}$ unless $e_{1 k}=e_{0}$ and $y_{1 k} \geq y_{0}$, in which case $t_{1 k}=t_{0}$ ) be a sequence such that $m_{1 k}=\left(e_{1 k}, y_{1 k}\right) \rightarrow m_{1}=\left(e_{1}, y_{1}\right)$. Since $m_{1} \notin J^{+}\left(m_{0}\right)$, it must be $y_{1}<y_{0}-\epsilon$, for a suitable $\epsilon>0$, indeed any event $m_{1}$ with $y_{1} \geq y_{0}$ is reached from $m_{0}$ by the future directed lightlike geodesic generated by $n$ starting from $m_{0}$. Since $y_{1 k} \rightarrow y_{1}<y_{0}-\epsilon$ it is $t_{1 k}>t_{0}$, thus $y_{0}+\mathcal{I}\left(e_{0}, e_{1 k}\right) \leq y_{1 k}<y_{0}-\epsilon$ in contradiction with the lower semi-continuity of $\mathcal{I}$.

A nice feature of the theorem is that it relates the causal simplicity of $(M, g)$ with properties of the classical action functional alone. There is no direct requirement on the time dependent metric $a_{t}$, 1 -form field $b_{t}$ and scalar field $\mathcal{U}(t, q)$. Roughly speaking (i.e. neglecting condition (i)), it shows that the property of existence of minimizers for the classical action is equivalent to the property of causal simplicity for Eisenhart's spacetime.

Thanks to the theorem it is quite easy to construct spacetimes which are not causally simple. As a matter of fact similar examples already appeared in the 
literature. In fact, after the discovery by Penrose 42 that pp-waves are not globally hyperbolic Ehrlich and Emch [14] showed that they are not even causally simple (for them $a_{a b}=\delta_{a b}, a, b=1,2, b_{t}=0$ and $\mathcal{U}$ is a suitable time dependent quadratic form in $q^{1}$ and $q^{2}$ ).

In their study of the general plane waves [17, 18, Flores and Sánchez prove (using only differential geometric tools) that if $a_{t}$ is independent of time, $(S, a)$ is a complete Riemannian manifold, $b_{t}=0$, and $\mathcal{U}$ has a subquadratic behavior at spatial infinity then, (a) the spacetime $(M, g)$ is globally hyperbolic [17, Theor. 4.1] (and hence causally simple), and (b) the action functional is bounded from below [17, Lemma 3.3]. From theorem 5.5. point (ii), it follows that under the same conditions the classical action functional admits a minimizer. We conclude that only by using tools from global Lorentzian geometry it is possible to prove results on the existence of minimizers that were previously obtained by using variational tools such as Ljusternik-Schnirelman and Morse theories 9.

Theorem [5.5 has an analog in the spacelike dimensional reduction case [37. Indeed, it can be shown that a Lorentzian direct product $M \times H$, with $(M, g)$ Lorentzian and $(H, h)$ Riemannian manifold, is causally simple if and only if the base spacetime $(M, g)$ is causally simple, the Lorentzian distance on the base is upper semicontinuous (the analog of condition (i)) and any two causally related events on $(M, g)$ at finite Lorentzian distance are connected by a maximizing geodesic (the analog of condition (ii)).

\section{Conclusions}

In the first part of the work it has been explained how Eisenhart's spacetime can be recovered from coordinate independent assumptions, and how this assumptions relate to the Bargmann structures. In the process a one-to-one correspondence between Newtonian frames and Abelian connections on suitable lightlike principal bundles, since now passed unnoticed, has been proved. It is expected to considerably simplify the presentation and study of Newton-Cartan theory.

In the second part a causal version of Eisenhart's theorem was proved and its connection with a Fermat type principle was clarified. The concept of light lift was introduced and exploited showing that the last coordinate of the light lift is in fact the classical action up to a constant. The tools introduced led us to recognize that the causal simplicity of Eisenhart's spacetime is almost equivalent to the property of the existence of minimizers for the associated classical action.

The bridge between relativistic and non-relativistic physics provided by the mathematics of lightlike dimensional reduction makes it possible to use the powerful tools from global Lorentzian geometry to attack, from a different perspective, some unsolved problems in classical mechanics. More on this subject will be done in subsequent works.

\section{Acknowledgements}

I thank M. Modugno for pointing out some references on Newton-Cartan theory and M. Sánchez for a careful reading of the manuscript. I also thank the referees for their useful criticisms. 


\section{Appendix: The relation with Newton-Cartan theory}

There is no (Newtonian frame independent) way of extending the metric (9) to a metric in the whole tangent space to $E$. Nevertheless, if $a^{a b}$ denotes the contravariant metric $a^{a b} a_{b c}=\delta_{c}^{a}$ then $a^{a b}$ can be naturally extended to a degenerate contravariant metric on the whole cotangent space of $E, a^{A B}, A, B=0,1, \ldots, d$, by setting $a^{A B} t_{, B}=0$. This contravariant metric $a^{-1}$ can also be obtained by pulling back the 1-forms from $E$ to $M$ and by applying to them $g^{-1}$, i.e. $a^{-1}=\pi_{*} g^{-1}$. A structure of this kind, in which a rank-d contravariant metric is annihilated by a 1 -form field $\eta=\psi \mathrm{d} t$ on a $d+1$ dimensional manifold is called a flat Galilei structure 29. The flat Galilei structure is of first order if $\mathrm{d} \eta=0$ on $E$, and in the spacetime $M$ it coincides with the Bargmann structure condition $\mathrm{d} n=0$.

If one tries to include an affine connection $\nabla^{(E)}$ on $E$ that preserves the contravariant metric and $\eta$, (i.e. the Galilei structure), $\nabla^{(E)} a^{-1}=0, \nabla^{(E)} \eta=0$, then a number of nice theorems arise 29. For instance, the affine connection is torsionless iff $\mathrm{d} \eta=0$. However, this condition does not determine the connection completely. Indeed, the torsionless connections turn out to be in one-to-one correspondence with the 2-forms on $E$. This set is restricted to the closed 2-forms if a particular Newtonian condition, that is a symmetry condition on the Riemann tensor, is imposed on the physical affine connections. Finally, the dynamical field equations $R_{A B}^{(E)}=4 \pi \rho \eta_{A} \eta_{B}$ for a given scalar matter density $\rho$ imply that the space section $S_{t}$ are Euclidean $a_{a b}=\delta_{a b}$, that the connection only depends on a potential $U$ in the usual way [39, Chapter 12], $\Gamma_{00}^{(E) a}=\partial_{a} U$, and that $U$ satisfies the Poisson equation $\triangle U=4 \pi \rho$. We see that two seemingly unrelated properties of the old Newtonian spacetime of classical mechanics, such as Newton's force law and the flatness of space are unified in the same geometrical framework.

Let us exploit the relation between the Newton-Cartan theory sketched above and the Bargmann structures 11, 12. The metric $g$ on $M$ determines an affine connection $\nabla$ which projects into an affine connection on $E$ as follows. Let $w$, be a vector field on $T E$, and let $W$ be any representative on $M, \pi_{*} W=w$. This last condition can be written $L_{n} W \propto n$, and implies from $L_{X} Y=[X, Y]=\nabla_{X} Y-\nabla_{Y} X$, and from the fact that $n$ is covariantly constant, $\nabla_{n} W \propto n$. As a consequence

$$
\nabla_{V+\alpha n}(W+\beta n)=\nabla_{V} W+\alpha \nabla_{n} W+n\left(\nabla_{V+\alpha n} \beta\right)
$$

has a projection independent of the fields $\alpha$ and $\beta$, and hence defines a connection $\nabla_{v}^{(E)} w=\pi_{*}\left(\nabla_{V} W\right)$ on $E$. It is easy to check that $\nabla^{(E)}$ preserves the Galilei structure. Moreover, it can be shown to be Newtonian [11. The Bargmann structure is then simpler than the Galilei structure since no Newtonian condition on the Galilei connection must be added. It appears to be extremely natural for the formulation of classical theories.

Newton-Cartan theory could prove important at the quantum level although very few works deal with the problem of formulating it. As it was pointed out by Kuchăr 28 the quantized Newton-Cartan theory would represent a face of the cube of theories (parametrized with $1 / c, G, \hbar$, and where classical mechanics corresponds to (000)), that is the face of equation $1 / c=0$, the other two known faces being Quantum Field Theory, i.e. $G=0$, and General Relativity, i.e. $\hbar=0$. The quantum theory of a particle on a curved Newton-Cartan background can be approached by means of path integral quantization methods or through geometric quantization strategies [40. Little is known on the quantization of the geometric fields, but it seems that they would 
be non-dynamical [28].

[1] V. I. Arnold. Mathematical methods of classical mechanics. Springer-Verlag, New York, 1978.

[2] J. K. Beem, P. E. Ehrlich, and K. L. Easley. Global Lorentzian Geometry. Marcel Dekker Inc., New York, 1996.

[3] J.K. Beem, P. E. Ehrlich, and S. Markvorsen. Timelike isometries and Killing fields. Geom. Dedicata, 26:247-258, 1988.

[4] I. M. Benn. Geodesics and Killing tensors in mechanics. J. Math. Phys., 47:022903, 2006.

[5] A. N. Bernal and M. Sánchez. Leibnizian, Galilean and Newtonian structures of spacetime. J. Math. Phys., 44:1129-1149, 2003.

[6] A. N. Bernal and M. Sánchez. Globally hyperbolic spacetimes can be defined as 'causal' instead of 'strongly causal'. Class. Quantum Grav., 24:745-749, 2007.

[7] G. A. Bliss. Lectures on the calculus of variations. The University of Chicago Press, Chicago, 1946.

[8] P. Bolle. On the Bolza problem. J. Differential Equations, 152:274-288, 1999.

[9] A. M. Candela, J. L. Flores, and M. Sánchez. A quadratic Bolza-type problem in a Riemannian manifold. J. Differential Equations, 193:196-211, 2003.

[10] L. Cesari. Optimization - Theory and applications. Springer-Verlag, New York, 1983.

[11] C. Duval, G. Burdet, H. P. Künzle, and M. Perrin. Bargmann structures and Newton-Cartan theory. Phys. Rev. D, 31:1841-1853, 1985.

[12] C. Duval, G. Gibbons, and P. Horváthy. Celestial mechanics, conformal structures, and gravitational waves. Phys. Rev. D, 43:3907-3922, 1991.

[13] J. Ehlers and W. Kundt. Exact solutions of the gravitational field equations, volume Gravitation: an introduction to current research, L. Witten (Ed.), chapter 2, pages 49-101. John Wiley \& Sons, New York, 1962.

[14] P. E. Ehrlich and G. G. Emch. Gravitational waves and causality. Rev. Math. Phys., 4:163-221, 1992.

[15] L. P. Eisenhart. Dynamical trajectories and geodesics. Ann. Math. (Ser 2), 30:591-606, 1929.

[16] I. Ekeland, N. Ghoussoub, and H. Tehrani. Multiple solutions for a classical problem in the calculus of variations. J. Differential Equations, 131:229-243, 1996.

[17] J. L. Flores and M. Sánchez. Causality and conjugate points in general plane waves. Class. Quantum Grav., 20:2275-2291, 2003.

[18] J. L. Flores and M. Sánchez. On the geometry of pp-wave type spacetimes. Lect. Notes Phys., 692:79-9, 2006.

[19] V.P. Frolov and I.D. Novikov. Black hole physics: Basic concepts and new developments. Kluwer Academic Publishers, Dordrecht, 1998.

[20] Y. Gliklikh. Global analysis in mathematical physics. Springer, New York, 1997.

[21] S. W. Hawking and G. F. R. Ellis. The Large Scale Structure of Space-Time. Cambridge University Press, Cambridge, 1973.

[22] E. Ihrig and D. K. Sen. Uniqueness of timelike Killing vector fields. Ann. Inst. H. Poincaré A, 23:297-301, 1975.

[23] A. D. Ioffe and V. M. Tihomirov. Theory of extremal problems. North-Holland Publishing Co., Amsterdam, 1979.

[24] P. Jordan, J. Ehlers, and R. Sachs. Beiträge zur Theorie der reinen Gravitationsstrahlung. Akad. Wiss. Mainz. Abh. Math. Naturwiss. Kl., 1:2, 1961.

[25] B. Julia and H. Nicolai. Null-Killing vector dimensional reduction and Galilean geometrodynamics. Nucl. Phys. B, 439:291-323, 1994.

[26] S. Kobayashi and K. Nomizu. Foundations of Differential Geometry, volume I of Interscience tracts in pure and applied mathematics. Interscience Publishers, New York, 1963.

[27] I. Kovner. Fermat principle in arbitrary gravitational fields. Astrophys. J., 351:114-120, 1990.

[28] K. Kuchăr. Gravitation, geometry, and nonrelativistic quantum theory. Phys. Rev. D, 22:12851299, 1980.

[29] H. P. Künzle. Galilei and Lorentz structures on space-time: comparison of the correspondig geometry and physics. Ann. Inst. H. Poincaré Phys. Theor., 17:337-362, 1972.

[30] H. P. Künzle and C. Duval. Relativistic and non-relativistic classical field theory on fivedimensional spacetime. Class. Quantum Grav., 3:957-973, 1986.

[31] A. Lichnerowicz. Théories relativistes de la gravitation et de l'électromagnetisme, Relativité Générale et théories unitaires. Masson, Paris, 1955.

[32] B. Lukács, Z. Perjés, and Á. Sebestyén. Null Killing vectors. J. Math. Phys., 22:1248-1253, 1981.

[33] R. Mañé. Global Variational Methods in Conservative Dynamics. IMPA, Rio de Janeiro, 1993. 
[34] J. E. Marsden and T. S. Ratiu. Introduction to Mechanics and Symmetry. Springer, New York, 1999.

[35] E. Minguzzi. On the existence of maximizing curves for the charged-particle action. Class. Quantum Grav., 20:4169-4175, 2003.

[36] E. Minguzzi. Classical aspects of lightlike dimensional reduction. Class. Quantum Grav., 23:7085-7110, 2006.

[37] E. Minguzzi. On the causal properties of warped product spacetimes. gr-qc/0612182, 2006.

[38] E. Minguzzi and M. Sánchez. The causal hierarchy of spacetimes. Cont. to Proc. of the ESI Semester 'Geometry of pseudo-Riemannian Manifolds with Application to Physics', ed. D. Alekseevsky and H. Baum (ESI, Vienna, Sept-Dec 2005) (European Mathematical Society Publishing House), gr-qc/0609119. To appear.

[39] C. W. Misner, K. S. Thorne, and J. A. Wheeler. Gravitation. Freeman, San Francisco, 1973.

[40] M. Modugno, C. Tejero, and R. Vitolo. Comparison between Geometric Quantisation and Covariant Quantum Mechanics, volume Proceedings of Lie Theory and Its Applications in Physics III, Clausthal 1999, ed. H.-D. Doebner, V.K. Dobrev and J. Hilgert. World Scientific, 2000.

[41] C. Nash and S. Sen. Topology and geometry for physicists. Academic Press, London, 1982.

[42] R. Penrose. A remarkable property of plane waves in general relativity. Rev. Mod. Phys., $37: 215-220,1965$.

[43] V. Perlick. On Fermat's principle in general relativity: I. the general case. Class. Quantum Grav., 7:1319-1331, 1990.

[44] M. Pettini. Geometrical hints for a nonperturbative approach to Hamiltonian dynamics. Phys. Rev. E, 47:828-850, 1993.

[45] R. Sachs. Gravitational waves in general relativity VI. The outgoing radiation condition. Proc. R. Soc. London A, 264:309-338, 1961.

[46] M. Szydlowski. The Eisenhart geometry as an alternative description of dynamics in terms of geodesics. Gen. Relativ. Gravit., 30:887-914, 1998.

[47] R. M. Wald. General Relativity. The University of Chicago Press, Chicago, 1984.

[48] A. Zeghib. On affine actions of Lie groups. Math. Zeitschrift, 227:245262, 1998. 